\title{
Ventral Tegmental Area GABA, glutamate, and glutamate-GABA neurons are heterogenous in their electrophysiological and pharmacological properties
}

\author{
Miranda-Barrientos Jorge ${ }^{1}$, Chambers Ian ${ }^{1}$, Mongia Smriti ${ }^{1}$, Liu Bing ${ }^{1}$, Wang Hui-Ling ${ }^{1}$, Mateo-Semidey Gabriel E ${ }^{1}$, \\ Margolis Elyssa $\mathrm{B}^{2}$, Zhang Shiliang ${ }^{3}$, Morales Marisela ${ }^{1 *}$ \\ ${ }^{1}$ Integrative Neuroscience Research Branch, National Institute on Drug Abuse, Baltimore, Maryland $21224 .{ }^{2}$ UCSF Weill \\ Institute of Neurosciences, Department of Neurology, University of California, San Francisco, San Francisco, CA 94158, \\ USA. ${ }^{3}$ Confocal and Electron Microscopy Core, National Institute on Drug Abuse, Baltimore, Maryland 21224
}

*Corresponding author: Dr. Marisela Morales, Integrative Neuroscience Research Branch, National Institute on Drug Abuse, 251 Bayview Blvd., Baltimore, Maryland 21224, MMORALES@intra.nida.nih.gov

Keywords: VTA, VTA-VGluT2 neurons, VTA-GABA neurons, glutamate-GABA neurons, mu opioid receptors

Impact Statement (15-30 words). Some physiological properties of VTA glutamate-releasing and glutamate-GABA coreleasing neurons are distinct from those of VTA GABA-releasing neurons. $\mu$-opioid receptor activation hyperpolarizes some VTA glutamate-releasing and some GABA-releasing neurons. 


\begin{abstract}
The ventral tegmental area (VTA) contains dopamine neurons intermixed with GABA-releasing (expressing vesicular GABA transporter, VGaT), glutamate-releasing (expressing vesicular glutamate transporter, VGluT2), and co-releasing (co-expressing VGaT and VGluT2) neurons. By delivering INTRSECT viral vectors into VTA of double vglut2Cre/vgat-Flp transgenic mice, we targeted specific VTA cell populations for ex vivo recordings. We found that VGluT2 ${ }^{+}$ $\mathrm{VGaT}^{-}$and $\mathrm{VGluT}^{+} \mathrm{VGaT}^{+}$neurons on average had relatively hyperpolarized resting membrane voltage, greater rheobase, and lower spontaneous firing frequency compared to VGluT2- VGaT $^{+}$neurons, suggesting that VTA glutamatereleasing and glutamate-GABA co-releasing neurons require stronger excitatory drive to fire than GABA-releasing neurons. In addition, we detected expression of Oprm1mRNA (encoding $\mu$ opioid receptors, MOR) in VGluT2 $2^{+} \mathrm{VGaT}^{-}$ and VGluT2- ${ }^{-} \mathrm{VGT}^{+}$neurons, and their hyperpolarization by the MOR agonist DAMGO. Collectively, we demonstrate the utility of the double transgenic mouse to access VTA glutamate, glutamate-GABA and GABA neurons, and show some electrophysiological heterogeneity among them.
\end{abstract}




\section{Introduction}

The ventral tegmental area (VTA) is a midbrain structure containing dopamine neurons that play a major role in motivated behaviors (Wise, 2004, Berridge et al., 2007, Bromberg-Martin et al., 2010). While it has classically been thought of as a dopaminergic structure, the VTA contains multiple types of neurons, including neurons that release GABA (expressing the synthesis enzyme glutamate decarboxylase, GAD, and the vesicular GABA transporter, VGaT) and neurons that release glutamate (expressing vesicular glutamate transporter type 2, VGluT2) (Yamaguchi et al., 2007). Moreover, we recently demonstrated that some VTA neurons release both glutamate and GABA (Root et al., 2014). These neurons express both VGluT2 and VGaT mRNA (VGluT2 ${ }^{+} \mathrm{VGaT}^{+}$neurons), whose proteins are distributed in separate pools of synaptic vesicles within a common axon terminal (Root et al., 2018). We have found a lateromedial gradient of distribution for $\mathrm{VGluT}^{+} \mathrm{VGaT}^{+}$neurons and those that release glutamate without GABA (VGluT2 ${ }^{+} \mathrm{VGaT}^{-}$neurons) or release GABA without glutamate (VGluT2 ${ }^{-} \mathrm{VGaT}^{+}$neurons) (Root et al., 2018).

The ex vivo electrophysiological properties of putative VTA dopamine neurons had been investigated for decades; the dopaminergic identification of VTA recorded neurons has been achieved by the immunocytochemical detection of tyrosine hydroxylase (TH) in rat (Margolis et al., 2006) or by in vivo labeling in transgenic mice (Khaliq \& Bean, 2010). Characterization of VTA GABA neurons in ex vivo recordings has been achieved by GAD protein and mRNA detection in recorded neurons in rat (Margolis et al., 2012) or by in vivo labeling in transgenic mice with constitutive or viral vectorinduced expression of green fluorescent protein (GFP) under the control of the GAD 2 (Tan et al., 2012) or VGaT (va Zessen et al., 2012) promoters. Ex vivo recordings of VTA glutamate neurons have been made in mice that constitutively express GFP under the control of the VGluT2 promoter (Hnasko et al., 2012). Importantly, these studies did not differentiate neurons that co-release glutamate and GABA $\left(\mathrm{VGluT}^{+} \mathrm{VGaT}^{+}\right.$) from those that are only glutamate-releasing $\left(\mathrm{VGluT}^{+} \mathrm{VGaT}^{-}\right)$or GABA-releasing $\left(\mathrm{VGluT}^{-} \mathrm{VGaT}^{+}\right.$), raising the possibility that some of the overlapping physiological properties reported in these groups specifically belong to this co-releasing population.

While existing transgenic mice allow in vivo tagging of the entire population of VTA neurons expressing VGluT2 or $\mathrm{VGaT}$, these transgenic lines are not suitable for the selective tagging of VGluT2 ${ }^{+} \mathrm{VGaT}^{+}, \mathrm{VGluT}^{+} \mathrm{VGaT}^{-}$, or VGluT2 ${ }^{-}$ $\mathrm{VGaT}^{+}$neurons. To overcome this limitation, we applied an intersectional approach to induce expression of enhanced YFP (eYFP) in the different classes of VTA neurons (Fenno et al., 2014). We found that some electrophysiological properties do vary across these different classes of VTA neurons, and determined that not only VGluT2 ${ }^{-} \mathrm{VGaT}^{+}$neurons, but also VGluT2 ${ }^{+} \mathrm{VGaT}^{-}$neurons, were postsynaptically inhibited by MOR activation. 


\section{Results}

Selective targeting of VTA VGluT2 ${ }^{+} \mathbf{V G a T}^{+}$, VGluT2 $^{+} \mathrm{VGaT}^{-}$and VGluT2 ${ }^{-} \mathrm{VGaT}^{+}$neurons. To tag $\mathrm{VGluT2}^{+} \mathrm{VGaT}^{+}$, $\mathrm{VGluT2}^{+} \mathrm{VGaT}^{-}$, and VGluT2 ${ }^{-} \mathrm{VGaT}^{+}$neurons, we generated vglut2-Cre/vgat-Flp mice by crossing vglut2-Cre mice with vgat-Flp mice (Figure 1A) and injected INTRSECT adeno associated viral (AAV) vectors into their VTA (Fenno et al., 2014). In different cohorts of vglut2-Cre/vgat-Flp mice, we injected AAV-C $\mathrm{ON}_{\mathrm{O}} / \mathrm{F}_{\mathrm{ON}}-\mathrm{eYFP}$ vectors (requiring Cre and Flp recombinases for eYFP expression) to target VGluT2 ${ }^{+} \mathrm{VGaT}^{+}$neurons; $\mathrm{AAV}-\mathrm{CoN}_{\mathrm{ON}} / \mathrm{F}_{\mathrm{OFF}}-\mathrm{eYFP}$ vectors (requiring the presence of Cre recombinase and absence of Flp recombinase for eYFP expression) to target VGluT2 ${ }^{+}$ $\mathrm{VGaT}^{-}$neurons; or AAV-C $\mathrm{CFF}_{\mathrm{F}} / \mathrm{F}_{\mathrm{ON}}-\mathrm{eYFP}$ vectors (requiring the absence of Cre recombinase and presence of Flp recombinase for eYFP expression) to target $\mathrm{VGluT2}^{-} \mathrm{VGaT}^{+}$neurons (Figure 1B-C).

After confirming VTA neuronal expression of eYFP in each cohort of mice (Figure 1D-F), we examined the mRNA expression of VGluT2 or VGaT within eYFP expressing neurons (Figure 1D-F). In the VTA of mice locally injected with AAV-C $\mathrm{CN}_{\mathrm{ON}} / \mathrm{F}_{\mathrm{ON}}-\mathrm{eYFP}$ vectors (to tag VGluT2 ${ }^{+} \mathrm{VGaT}^{+}$neurons) (Figure 1D), we found that within the total population of eYFP expressing neurons (1,647 neurons, 3 mice; Figure $1 \mathrm{G})$, approximately $90 \%$ expressed both VGluT2 and VGaT mRNAs ( $87.3 \% \pm 2.4 \% ; 1,441 / 1,647) ; 6 \%$ expressed only VGluT2 mRNA $(5.6 \% \pm 1.6 \% ; 88 / 1,647$ neurons), close to $5 \%$ expressed only VGaT mRNA $(5.2 \% \pm 2.0 \% ; 85 / 1,647$, Figure $1 \mathrm{G})$, and rarely lacked both VGluT2 and VGaT mRNAs $(1.93 \% \pm 0.35 \%$; $33 / 1647)$. In the VTA of mice locally injected with AAV-C $\mathrm{CoN} / \mathrm{F}_{\text {OFF- }}$ eYFP vectors (to tag VGluT2 ${ }^{+}$VGAT $^{-}$neurons) (Figure 1E), we found that within the total population of eYFP neurons (2,804 neurons, 3 mice; Figure 1G) more than $90 \%$ expressed VGluT2 mRNA $(93.1 \% \pm 2.8 \%$, $2,563 / 2,804)$, none expressed VGaT mRNA alone, they rarely expressed VGluT2 mRNA together with VGaT mRNA $(2.1 \% \pm 0.9 \%, 76 / 2,804)$, and a small number lacked both VGluT2 or VGaT mRNAs $(4.8 \% \pm 2.0 \%, 165 / 2,804)$. In the VTA of mice locally injected with AAV-C $\mathrm{C}_{\mathrm{OFF}} / \mathrm{F}_{\mathrm{ON}}$-eYFP vectors (to tag VGluT2 ${ }^{-} \mathrm{VGaT}^{+}$neurons) (Figure 1F), we found that within the total population of eYFP neurons, close to $85 \%$ expressed VGaT mRNA $(84.7 \% \pm 2.1 \%$, $1,904 / 2,228$ neurons, 3 mice) (Figure 1F, G), rarely expressed VGluT2 mRNA $(2.3 \% \pm 1.1 \%, 41 / 2,228$ neurons) (Figure $1 \mathrm{G})$, infrequently had VGaT mRNA together with VGluT2 mRNA (4.4\% $\pm 1.4 \%$; 91/2,228 neurons) (Figure 1G), and a small number lacked both VGluT2 and VGaT mRNAs $(8.6 \% \pm 0.5 \%, 192 / 2,228$ neurons) (Figure 1G). Collectively, these findings indicate that using vglut2-Cre/vgat-Flp mice in combination with the tested INTRSECT viral vectors for the selective tagging of the three classes of VTA neurons generates very few false positive classifications.

Intrinsic properties of VTA VGluT2 ${ }^{+} \mathrm{VGaT}^{+}$, $\mathrm{VGluT2}^{+} \mathrm{VGaT}^{-}$and $\mathrm{VGluT2}^{-} \mathrm{VGaT}^{+}$neurons. Next we determined the spontaneous action potential (AP) activity of the three classes of neurons with cell attached recordings in horizontal brain slices. We detected spontaneous activity in $46.2 \%$ of the VGluT2 ${ }^{+} \mathrm{VGaT}^{+}$neurons $(24 / 52$ neurons, 28 mice), $52.6 \%$ of the VGluT2 ${ }^{+} \mathrm{VGaT}^{-}$neurons (24/49 neurons, 27 mice) and $70.7 \%$ of the $\mathrm{VGluT}^{-} \mathrm{VGaT}^{+}$neurons (29/41 neurons, 19 mice) (Figure 2C). Within the spontaneously active neurons ( 77 neurons), we found that the mean firing frequency was lower in $\mathrm{VGluT}^{+} \mathrm{VGaT}^{+}$and $\mathrm{VGluT}^{+} \mathrm{VGaT}^{-}$neurons than in $\mathrm{VGluT}^{-} \mathrm{VGaT}^{+}$neurons $(3.4 \pm$ $0.7 \mathrm{~Hz}$ for VGluT2 ${ }^{+} \mathrm{VGaT}^{+}, \mathrm{n}=24$ neurons, 20 mice; $3.5 \pm 0.7 \mathrm{~Hz}$ for $\mathrm{VGluT2}^{+} \mathrm{VGaT}^{-}, \mathrm{n}=24$ neurons, 18 mice; and $8.3 \pm 2.1 \mathrm{~Hz}$ for VGluT2 ${ }^{-} \mathrm{VGaT}^{+} \mathrm{n}=29$ neurons, 14 mice) (Figure $2 \mathrm{~B}, \mathrm{D}$ ). Together these findings indicate that more VGluT2 ${ }^{-} \mathrm{VGaT}^{+}$neurons fire spontaneously, and on average they fire faster, than $\mathrm{VGluT}^{+} \mathrm{VGaT}^{+}$or $\mathrm{VGluT}^{+} \mathrm{VGaT}^{-}$ neurons. Next, we analyzed the extracellular AP durations from these neurons and found that $\mathrm{VGluT2}^{+} \mathrm{VGaT}^{+}$ neurons have similar AP durations to VGluT2- $\mathrm{VGaT}^{+}$neurons, and both of these phenotypes have shorter AP durations than VGluT2 ${ }^{+} \mathrm{VGaT}^{-}$neurons $\left(1.3 \pm 0.1 \mathrm{~ms}\right.$ for VGluT2 ${ }^{+} \mathrm{VGaT}^{+}, \mathrm{n}=24$ neurons, 20 mice; $1.7 \pm 0.1 \mathrm{~ms}$ for VGluT2 $^{+}$VGaT $^{-}, \mathrm{n}=24$ neurons, 18 mice; and $1.2 \pm 0.1 \mathrm{~ms}$ for VGluT2 ${ }^{-} \mathrm{VGaT}^{+} \mathrm{n}=29$ neurons, 14 mice) (Figure $2 \mathrm{~F}, \mathrm{G})$. Because VTA neurons are known to have pacemaker activity as well as burst firing patterns, we also evaluated the regularity of firing. We analyzed the coefficient of variation (CV) of inter-spike intervals (ISIs), and found that both VGluT2 ${ }^{+} \mathrm{VGaT}^{+}$and VGluT2 ${ }^{+} \mathrm{VGaT}^{-}$neurons had higher CVs of ISIs compared to VGluT2- $\mathrm{VGaT}^{+}$neurons $\left(48.5 \pm 6.00\right.$ for $\mathrm{VGluT}^{+} \mathrm{VGaT}^{+}, 41.8 \pm 6.5$ for $\mathrm{VGluT}^{+} \mathrm{VGaT}^{-}$, and $37.2 \pm 4.9$ for $\left.\mathrm{VGluT}^{-} \mathrm{VGaT}^{+}\right)$(Figure 2E). These findings indicate that the spontaneous firing of $\mathrm{VGluT}^{+} \mathrm{VGaT}^{+}$and $\mathrm{VGluT2}^{+} \mathrm{VGaT}^{-}$neurons is more irregular than that of VGluT2 $2^{-} \mathrm{VGaT}^{+}$neurons.

Next, using whole-cell recordings, we examined intrinsic electrophysiological properties of these three classes of VTA neurons. We found that the resting membrane potential was $-68.0 \pm 1.2 \mathrm{mV}$ for VGluT2 $2^{+} \mathrm{VGaT}^{+}$neurons $(\mathrm{n}=52$ neurons, 27 mice), $-64.6 \pm 0.9 \mathrm{mV}$ for VGluT2 ${ }^{+} \mathrm{VGaT}^{-}$neurons $(\mathrm{n}=49$ neurons, 27 mice), and $-59.6 \pm 0.9 \mathrm{mV}$ for VGluT2 $^{-} \mathrm{VGaT}^{+}$neurons ( $\mathrm{n}=41$ neurons, 19 mice). We detected the greatest rheobase in $\mathrm{VGluT2}^{+} \mathrm{VGaT}^{-}$neurons (37.3 $\pm 5.6 \mathrm{pA}, \mathrm{n}=48$ neurons, 27 mice), followed by VGluT2 ${ }^{+} \mathrm{VGaT}^{+}$neurons $(29.0 \pm 5.5 \mathrm{pA}, \mathrm{n}=52$ neurons, 28 mice), 
and the lowest rheobase in VGluT2- $\mathrm{VGaT}^{+}$neurons $(14.9 \pm 2.9 \mathrm{pA}, \mathrm{n}=41,19$ mice) (Table 1$)$. Collectively these findings indicate that VTA neurons expressing VGluT2, with or without VGaT, are less excitable than VTA neurons lacking VGluT2, and the VGluT2 ${ }^{+} \mathrm{VGaT}^{-}$neurons are the least excitable among the 3 classes. Across the three classes of VTA neurons, there were no differences in membrane capacitance, membrane resistance, membrane time constant, or AP threshold. However, in line with our cell attached data, we found that VGluT2 ${ }^{+} \mathrm{VGaT}^{-}$neurons have longer duration APs than VGluT2 ${ }^{+} \mathrm{VGaT}^{+}$and VGluT2 ${ }^{-} \mathrm{VGaT}^{+}$neurons $\left(2.2 \pm 0.1 \mathrm{~ms}\right.$ for $\mathrm{VGluT}^{+} \mathrm{VGaT}^{+}, \mathrm{n}=52$ neurons, mice; $2.9 \pm 0.2 \mathrm{~ms}$ for $\mathrm{VGluT2}^{+} \mathrm{VGaT}^{-}, \mathrm{n}=48$ neurons, 27 mice and $1.8 \pm 0.1 \mathrm{~ms}$ for $\mathrm{VGluT2}^{-} \mathrm{VGaT}^{+}, \mathrm{n}=41 \mathrm{neurons}, 19$ mice) (Table 1).

Given that hyperpolarization-activated cation currents $\left(I_{\mathrm{h}}\right)$ are present in both dopamine and non-dopamine neurons (Jones and Kauer, 1999; Margolis et al., 2006; Margolis et al., 2012; Hnasko et al., 2012), we tested the three classes of VTA neurons for $I_{\mathrm{h}}$. We detected $I_{\mathrm{h}}$ in $46.2 \%$ of VGluT2 ${ }^{+} \mathrm{VGaT}^{+}$neurons (24/52 neurons, 28 mice), 53.3\% of VGluT2 $^{+}$VGaT $^{-}$neurons (24/45 neurons from 24 mice) and $92 \%$ of VGluT2- VGaT $^{+}$neurons (35/38 neurons, 18 mice) (Figure 3B). While there was a wide range of $I_{\mathrm{h}}$ magnitude among each cell type, the mean $I_{\mathrm{h}}$ magnitude was smaller in $\mathrm{VGluT}^{+} \mathrm{VGaT}^{+}$neurons compared to $\mathrm{VGluT2}^{+} \mathrm{VGaT}^{-}$neurons or $\mathrm{VGluT2}^{-} \mathrm{VGaT}^{+}$neurons $(22.4 \pm 3.2 \mathrm{pA}$ for VGluT2 $^{+} \mathrm{VGaT}^{+}, 56.8 \pm 12.2 \mathrm{pA}$ for $\mathrm{VGluT}^{+} \mathrm{VGaT}^{-}$, and $48.3 \pm 8.7 \mathrm{pA}$ for $\mathrm{VGluT2}^{-} \mathrm{VGaT}^{+}$) (Figure $3 \mathrm{~A}, \mathrm{C}$ ). We found that regardless of the neuronal cell type, the neurons with larger $I_{\mathrm{h}}$ magnitudes were located in the lateral VTA and those with low amplitudes were in the medial VTA. These findings support a VTA latero-medial neuronal topography among dopamine and non-dopamine neurons.

\section{Stimulated firing patterns of VTA VGluT2 ${ }^{+}$VGaT $^{+}$, VGluT2 $^{+}$VGaT $^{-}$and VGluT2- ${ }^{-}$VaT $^{+}$neurons. We next} examined the stimulated firing patterns of these three classes of neurons by inducing AP firing in current clamp with a series of depolarizing current steps (500 ms, 10-150 pA).

We found that most $\mathrm{VGluT2}^{+} \mathrm{VGaT}^{+}$neurons (41/48 neurons, 24 mice) fired APs with short latency from initiation of the current pulse (159 $\pm 14 \mathrm{~ms}$; Figure 4A-B, D). A small group of neurons responded to the injected current with delayed firing (7/48 neurons, 6 mice; $383 \pm 47 \mathrm{~ms}$; Figure 4A-B, D), displaying a slow depolarizing ramp prior to the first AP (Figure 4A). We found that the rheobase was generally greater in VGluT2 ${ }^{+} \mathrm{VGaT}^{+}$neurons with long latency $(101.4 \pm$ $24.8 \mathrm{pA})$ than in those with short latency $(17.8 \pm 2.5 \mathrm{pA}$; Figure $4 \mathrm{C})$. We observed that current injections below $100 \mathrm{pA}$ produced depolarization block in some of the short latency neurons (14/41 neurons, 8 mice; Figure 4D). Across all short latency VGluT2 ${ }^{+} \mathrm{VGaT}^{+}$neurons (41 neurons), more than half of them fired APs continuously during the entire current injection (27/41 neurons, 18 mice; Figure 4D). Furthermore, many of these neurons with continuous AP firing showed frequency adaptation (being more evident at currents above $100 \mathrm{pA} ; 18 / 27$ neurons, 12 mice; Figure 4D), with the ISI increasing after each AP (Figure 4-figure supplement 1), but others either lacked or had minimal frequency adaptation (9/27 neurons, 7 mice; Figure 4-figure supplement 1$)$ that permitted higher firing rates $(38.7 \pm 3.2 \mathrm{~Hz}$ sustained firing with adaptation; $84.2 \pm 5.7 \mathrm{~Hz}$ for sustained firing without adaptation). VGluT2 ${ }^{+} \mathrm{VGaT}^{-}$neurons were similar to VGluT2 $\mathrm{VGaT}^{+}$neurons, with short latency AP firing in response to injected current (29/45 neurons, 22 mice; $122 \pm 16 \mathrm{~ms}$ latency) and fewer long latency responses with a depolarizing ramp leading to firing (16/45 neurons, 13 mice ; $462 \pm 11$ ms latency; Figure 4A-B, D). Among the neurons with depolarizing ramp responses $\left(\mathrm{VGluT2}^{+} \mathrm{VGaT}^{+}\right.$and $\mathrm{VGluT}^{+}$ VGaT $^{-}$neurons), we detected A-type $\mathrm{K}^{+}$currents $\left(I_{\mathrm{A}}\right)$, which were blocked by the $I_{\mathrm{A}}$ blocker 4-Aminopyridine (4-AP; 2 $\mathrm{mM}$ ) (Figure 4-figure supplement 2A-B). In addition, we found that 4-AP application decreased AP firing latency (4 VGluT2 $^{+}$VGaT $^{+}$neurons and 4 VGluT2 $^{+}$VGaT $^{-}$neurons, 8 mice; Baseline $=458 \pm 7 \mathrm{~ms}, 4-\mathrm{AP}=192 \pm 29 \mathrm{~ms}$; Figure 4figure supplement $2 \mathrm{~F})$, and increased the total number of APs fired during an input/output curve (10-150 pA, $500 \mathrm{~ms})(4$ VGluT2 $^{+} \mathrm{VGaT}^{+}$neurons and $4 \mathrm{VGluT}^{+} \mathrm{VGaT}^{-}$neurons, 8 mice; Baseline $=52.6 \pm 17.8,4-\mathrm{AP}=101 \pm 24.3$; Figure 4figure supplement $2 \mathrm{C}, \mathrm{G})$. Compared to any neurons expressing VGluT2 (VGaT ${ }^{+}$or $\left.\mathrm{VGaT}^{-}\right)$, we found that all recorded VGluT2 $^{-} \mathrm{VGaT}^{+}$neurons had short latency AP firing responses to injected depolarizing current steps (38 neurons, 18 mice, $127.7 \pm 13.5 \mathrm{~ms}$ latency; Figure 4A-B, D), and a subset showed frequency adaptation (22/38 neurons, 15 mice) (Figure 4D). Collectively, these results demonstrate that both glutamate-GABA co-releasing $\left(\mathrm{VGluT2}^{+} \mathrm{VGaT}^{+}\right.$) and glutamate-releasing $\left(\mathrm{VGluT}^{+} \mathrm{VGaT}^{-}\right)$neurons are more heterogeneous in their firing properties than GABA-releasing (VGluT2- VGaT $^{+}$) neurons.

Given that previous studies have shown hyperpolarization-induced rebound burst firing in a subset of VTA dopamine and non-dopamine neurons mediated by $I_{\mathrm{h}}$ (Tateno and Robinson, 2011) or T-type calcium channels (Tracy et al., 2018, Woodward et al., 2019), we next tested the extent to which rebound firing occurs in the three classes of VTA neurons. After holding the resting membrane potential at $-100 \mathrm{mV}$ for $1 \mathrm{sec}$ and then releasing that clamp, approximately $25 \%$ of 
VGluT2 $^{+} \mathrm{VGaT}^{+}$neurons showed rebound firing with short bursts of 2-4 APs (12/48 neurons, 10 mice; Figure 5), and this response was stable over repeated trials (Figure 5-suplement figure 1). In some of these neurons we tested if $I_{\mathrm{h}}$ blocker ZD 7288 stopped rebound firing, and found that it did in 2/4 neurons. In a different set of neurons we tested if mibefradil blocked the rebound firing, and found that it did in $3 / 5$ neurons by the $I_{\mathrm{h}}$ blocker ZD 7288 (2/4 tested neurons) while in others it was blocked by T-type calcium channel blocker (Mibefradil; 3/5 tested neurons). Furthermore, we observed that $35 \%$ of VGluT2 ${ }^{+} \mathrm{VGaT}^{-}$neurons $(16 / 45$ neurons, 11 mice) had rebound firing that was blocked by ZD 7288 (7/7 tested neurons) or Mibefradil (2/4 tested neurons) (Figure 5). In contrast, we found that half (20/38 neurons, 14 mice) of the VGluT2 ${ }^{-} \mathrm{VGaT}^{+}$neurons showed rebound firing, which was blocked by ZD 7288 (5/6 tested neurons) or Mibefradil (2/5 tested neurons) (Figure 5). These findings demonstrated that some of the glutamateGABA co-releasing neurons, glutamate-releasing and GABA-releasing neurons have rebound firing mediated by either $I_{\mathrm{h}}$ or T-type calcium channels.

VTA neuronal clustering by electrophysiological properties. We next evaluated the extent to which the electrophysiological properties described above grouped together by applying a cluster analysis based on K-mean clustering and similarity parametric (Pearson's correlation coefficient) on data obtained from the firing properties and intrinsic properties (Figure 6A). Based on the cluster analysis, we found 4 clusters of neurons with distinctive firing features, but these clusters did not uniquely correspond to any of our three neurotransmitter phenotypes (Figure 6B). For the 131 clustered neurons, $36.6 \%$ (48/131 neurons, 33 mice) grouped in cluster $1,32.1 \%(42 / 131$ neurons, 31 mice) in cluster 2, 13.7\% (18/131 neurons, 11 mice) in cluster 3, and 17.6\% (23/131 neurons, 20 mice) in cluster 4 (Figure 6B).

In cluster 1 (48 neurons), 39.5\% were VGluT2 ${ }^{+} \mathrm{VGaT}^{+}$(19/48 neurons, 12 mice), $18.8 \%$ were VGluT2 ${ }^{+}$VGaT $^{-}(9 / 48$ neurons, 7 mice), and $41.6 \%$ were $\mathrm{VGluT}^{-} \mathrm{VGaT}^{+}$(20/48 neurons, 15 mice) (Figure 6B). Neurons grouped within the cluster 1 were characterized by a sustained AP firing with marked adaptation $(46.5 \pm 2.8 \mathrm{~Hz}$ maximal AP frequency, Figure $5 \mathrm{H}$ ), and depolarized membrane potential $(-61.9 \pm 0.8 \mathrm{mV}$; Figure $6 \mathrm{E})$. We found that $75 \%$ of the neurons within this cluster were spontaneously active during cell attached recordings (36/48 neurons, 29 mice), representing the highest percentage of spontaneously active neurons among the four different clusters. In cluster $2,21.4 \%$ were $\mathrm{VGluT}^{+} \mathrm{VGaT}^{+}$ (9/42 neurons, 8 mice), $35.7 \%$ were VGluT2 ${ }^{+} \mathrm{VGaT}^{-}$(15/42 neurons, 12 mice), and $42.8 \%$ were $\mathrm{VGluT}^{-} \mathrm{VGaT}^{+}$ neurons (18/42 neurons, 12 mice) (Figure 6B). The neurons in this cluster had small rheobase (11.6 $\pm 1.4 \mathrm{pA}$; Figure 6F), short latency AP firing in response to depolarizing current steps ( $97 \pm 10 \mathrm{~ms}$ Figure $6 \mathrm{G})$, and sustained firing with minimal frequency adaptation in response to current steps $(76.1 \pm 3.4 \mathrm{~Hz}$; Figure $6 \mathrm{H})$. We found that $69 \%(29 / 42$ neurons, 23 mice) of the neurons in this cluster showed rebound firing after release from clamp induced hyperpolarization. In cluster $3,72.2 \%$ were VGluT2 ${ }^{+} \mathrm{VGaT}^{+}(13 / 18$ neurons, 8 mice $)$ and $27.8 \%$ were VGluT2 ${ }^{+}$VGaT $^{-}$neurons $(5 / 18$ neurons, 4 mice; Figure 6B). Neurons grouped in this cluster exhibited rapid depolarization block during low magnitude depolarizing current steps (Figure 6C, D). In cluster 4, 30.4\% (5/23 neurons from 5 mice) were $\mathrm{VGluT}^{+} \mathrm{VGaT}^{+}$and $69.5 \%$ were VGluT2 $^{+}$VGaT $^{-}$neurons $\left(16 / 23\right.$ neurons, 14 mice). No VGluT2 ${ }^{-} \mathrm{VGaT}^{+}$neurons were classified to this cluster. Neurons in cluster 4 had larger rheobase $(83.9 \pm 11.1 \mathrm{pA}$; Figure $6 \mathrm{~F})$, long latencies to AP firing in response to injected current steps $(452 \pm 11 \mathrm{~ms}$; Figure $6 \mathrm{G})$, a depolarizing ramp before the onset of AP firing during current steps (Figure 6C-E), and included some neurons with $I_{\mathrm{A}}$ (Supplementary Figure 2C-G). We found that all neurons within cluster 4 were quiescent during cell attached recordings.

Next, we determined whether the 4 identified clusters of physiological properties had specific topography within the VTA by mapping the distribution of neurons that we filled with biocytin after recordings. We found cluster 1 neurons in ventral and middle VTA, with a dorsal to ventral, and lateral to medial increasing gradient of distribution (Figure 7B). We frequently observed cluster 2 neurons in middle VTA concentrated more medially (Figure 7C), cluster 3 neurons in the ventral and middle VTA also enriched medially (Figure 7D) and cluster 4 neurons in the middle and dorsal VTA confined to the most medial part of the VTA (Figure 7E). These findings indicate that VGluT2 ${ }^{+} \mathrm{VGaT}^{+}, \mathrm{VGluT}^{+} \mathrm{VGaT}^{-}$and VGluT2 ${ }^{-} \mathrm{VGaT}^{+}$neurons have a topographic organization more related to shared electrophysiological properties rather than to the neurotransmitter that they release.

$\mu$-opioid receptors (MORs) are present in VTA GABA-releasing and glutamate-releasing neurons. Given that previous electrophysiological studies have demonstrated the presence of MORs in a subset of VTA GABA releasing neurons (Margolis et al., 2012), and anatomical studies have documented expression of Oprm1 mRNA in VTA VGluT2 neurons (Kudo et al., 2014), we systematically analyzed Oprm1 mRNA expression in VTA neurons that express VGluT2 mRNA and VGaT mRNA. We detected Oprm1 mRNA in VGluT2 ${ }^{+}$VGaT $^{-}$(Figure 8A), VGluT2- VGaT $^{+}$ 
(Figure $8 \mathrm{~B}$ ) and $\mathrm{VGluT}^{+} \mathrm{VGaT}^{+}$neurons in coronal mouse sections (Figure $8 \mathrm{C}$ ). We determined that within the total population of VTA neurons expressing Oprm1 mRNA (1,718 neurons, 3 mice), almost 20\% expressed VGluT2 mRNA without VGaT mRNA $(19.45 \% \pm 0.9 \%$; 336/1,718 neurons), $78 \%$ expressed VGaT mRNA without VGluT2 mRNA $(78 \% \pm 0.9 \% ; 1,337 / 1,718$ neurons $)$, and a small number co-expressed VGluT2 mRNA and VGaT mRNA $(2.5 \% \pm 0.2 \% ; 43 / 1,718$ neurons) (Figure $8 \mathrm{D})$. These findings demonstrated that GABA-releasing and glutamatereleasing neurons are two major classes of VTA neurons with the capability to synthesize MORs.

Next, we tested both VGluT2 ${ }^{+} \mathrm{VGaT}^{-}$and $\mathrm{VGluT}^{-} \mathrm{VGaT}^{+}$neurons for responses to the MOR selective agonist DAMGO $(1 \mu \mathrm{M})$. We detected DAMGO induced hyperpolarizations in a subset of VGluT2 ${ }^{+} \mathrm{VGaT}^{-}$neurons $(-7.1 \pm 1.7$ $\mathrm{mV} ;-64.3 \pm 2.7 \mathrm{mV}$ baseline, $-71.4 \pm 3.2 \mathrm{mV}$ DAMGO, $\mathrm{n}=7$ out of 17 tested neurons, 14 mice) (Figure $7 \mathrm{~F}-\mathrm{G}$ ). Hyperpolarizations were also observed in the presence of the $\mathrm{GABA}_{\mathrm{A}}$ receptor antagonist (Bicuculline, $\left.10 \mu \mathrm{M}\right)$ ( Figure 8supplement figure 1). Application of the MOR selective antagonist CTAP $(1 \mu \mathrm{M})$ reversed the DAMGO-induced hyperpolarizations $(-73.3 \pm 3.1 \mathrm{mV}$ baseline, $-84.5 \pm 2.7 \mathrm{mV}$ DAMGO, $-72.5 \pm 3.4 \mathrm{mV}$ for DAMGO + CTAP, 5 tested neurons) (Figure $7 \mathrm{H}-\mathrm{I}$ ). Similarly, we detected DAMGO induced hyperpolarizations in just over half of VGluT2- $\mathrm{VGaT}^{+}$ tested neurons $(-7.7 \pm 1.3 \mathrm{mV} ;-64.1 \pm 1.9 \mathrm{mV}$ baseline, $-71.8 \pm 2.1 \mathrm{mV}$ DAMGO, $\mathrm{n}=10$ out of 18 tested neurons, 15 mice) (Figure 7 F-G), and these were also reversed by CTAP (-58.9 \pm 1.0 baseline, $-64.9 \pm 1.2$ DAMGO, $-60.5 \pm 1.2$ DAMGO + CTAP, 6 tested neurons) (Figure $7 \mathrm{H}-\mathrm{I})$. Collectively, these findings indicate that a subset of VTA neurons that release either GABA or glutamate express functional MOR in their somatodendritic region, the activation of which results in their hyperpolarization. 


\section{Discussion}

The VTA has historically been considered a dopamine brain structure, and the properties of these dopamine neurons have been intensively investigated for decades. However, the VTA has three additional major classes of neurons: GABAreleasing (VGluT2 ${ }^{-} \mathrm{VGaT}^{+}$), glutamate-releasing $\left(\mathrm{VGluT}^{+} \mathrm{VGaT}^{-}\right)$and glutamate-GABA co-releasing $\left(\mathrm{VGluT}^{+} \mathrm{VGaT}^{+}\right.$) neurons, whose physiological properties were unclear. To specifically study these three classes of neurons, we selectively tagged each class by in vivo expression of eYFP after intra-VTA delivery of INTRSECT viral vectors $\left(\mathrm{C}_{\mathrm{ON}} / \mathrm{F}_{\mathrm{ON}}\right.$, $\mathrm{C}_{\mathrm{ON}} / \mathrm{F}_{\mathrm{OFF}}$ or $\left.\mathrm{C}_{\mathrm{OFF}} / \mathrm{F}_{\mathrm{ON}}\right)$ in different cohorts of double transgenic vglut2-Cre/vgat-Flp mice. We validated the selective expression of eYFP in each of the three targeted classes of VTA neurons by demonstrating that: (1) most of the VTA transduced neurons with $\mathrm{CON}_{\mathrm{ON}} / \mathrm{F}_{\mathrm{ON}}$ viral vector (for targeting glutamate-GABA co-releasing neurons) co-expressed VGluT2 and VGaT mRNAs, (2) those transduced with $\mathrm{C}_{\mathrm{ON}} / \mathrm{F}_{\mathrm{OFF}}$ viral vector (for targeting glutamate-releasing neurons) expressed VGluT2 mRNA without VGaT mRNA, and (3) those transduced with $\mathrm{C}_{\mathrm{OFF}} / \mathrm{F}_{\mathrm{ON}}$ viral vector (for targeting GABA-releasing neurons) expressed VGaT mRNA without VGluT2 mRNA. Using ex vivo VTA recordings of the three classes of transfected neurons, we found that both glutamate-releasing and glutamate-GABA co-releasing neurons have lower excitability and lower basal firing activity than GABA-releasing neurons. In addition, while we observed diversity in the depolarization-induced firing patterns of glutamate-releasing and glutamate-GABA co-releasing neurons, the responses among GABA-releasing neurons were more uniform. We also demonstrated that whereas most of the VTA neurons containing the $\mu$ opioid receptors (MORs) are GABA-releasing neurons, $40 \%$ of glutamate-releasing neurons were also hyperpolarized by MOR activation. Collectively, we provide evidence that: (1) the VTA neuronal firing of both glutamate-releasing and glutamate-GABA co-releasing neurons require a stronger excitatory input to fire than GABAreleasing neurons, (2) the ionic channel composition is likely to be more diverse among glutamate-releasing and glutamate-GABA co-releasing neurons than among GABA-releasing neurons, and (3) postsynaptic MOR activation inhibits the activity of both GABA-releasing and glutamate-releasing VTA neurons.

It is well documented that $I_{\mathrm{h}}$ is present in both dopamine and non-dopamine neurons (Jones and Kauer, 1999; Margolis et al., 2006), including neurons expressing GAD (Chieng et al., 2011; Margolis et al., 2012; Ntamati et al., 2018), VGaT (Woodward et al., 2019) or VGluT2 (Hnasko et al., 2012). We extended these observations by showing that $I_{\mathrm{h}}$ is present in less than half of the glutamate-GABA co-releasing neurons, about half of the glutamate-releasing neurons and in more than $90 \%$ of the GABA-releasing neurons. However, the meane $I_{\mathrm{h}}$ magnitude is smallest in glutamate-GABA co-releasing neurons, followed by GABA-releasing neurons, and largest in glutamate-releasing neurons. We found that regardless of the neuronal cell type, the neurons with the greatest $I_{\mathrm{h}}$ magnitudes were located in the lateral VTA, and those with low amplitudes were in the medial VTA. These findings are consistent with the VTA latero-medial neuronal heterogeneity observed in dopamine neurons (Li X et al.,2013, Morales and Margolis 2017) and show it is a property shared by all classes of VTA neurons. While the molecular bases underlying differences in $I_{\mathrm{h}}$ magnitude among VTA neurons remains to be determined, one possibility is differential levels of expression of any of the four hyperpolarization-activated cyclic nucleotide-gated channel (HCN1-4) subunits that generate $I_{\mathrm{h}}$ and whose transcripts have been detected in the VTA (Monteggia et al., 2000). As an alternative, differences in $I_{\mathrm{h}}$ magnitude may reflect differential neuronal distribution of the HCN channels across neuronal compartments, as the HCN subunits have been detected in the plasma membrane of cell bodies, dendrites, or axons (Notomi and Shigemoto, 2004).

While our findings on the intrinsic properties of VTA neurons suggest that stronger excitatory drive is required for firing in VTA glutamate-releasing and glutamate-GABA co-releasing neurons compared to VTA GABA-releasing neurons, it remains to be determined which glutamatergic sources target each class of VTA neuron. Ultrastructural and electrophysiological reports indicate that VTA neurons expressing either GAD or VGaT receive excitatory input from different brain areas. For instance, pioneer ultrastructural studies showed that VTA GABA-neurons (expressing GAD) receive asymmetric (excitatory-type) synapses from axon terminals whose neurons originate in the lateral habenula (Olmelchenko et al., 2009), medial prefrontal cortex (Carr and Sesack, 2000), periaqueductal grey (Olmelchenko and Sesack, 2010), and bed nucleus of the stria terminalis (Kudo et al., 2012). Furthermore, recent findings utilizing optogenetics and VTA slice electrophysiology have shown that the firing of GABA neurons (expressing VGaT or GAD) is evoked by exciting glutamatergic inputs (expressing VGluT2) from lateral hypothalamus neurons (Nieh et al., 2015), superior colliculus neurons (Zhou et al., 2019) or periaqueductal grey neurons (Ntamati et al., 2018). Moreover, a circuitry-based study on input from the periaqueductal grey to VTA showed that periaqueductal glutamatergic neurons preferentially target the GAD neurons with large $I_{\mathrm{h}}$ (Ntamati et al., 2018). Though it is possible that some of the GABA neurons identified in these prior studies were in fact glutamate-GABA co-releasing neurons, information on glutamatergic afferents to VTA glutamatergic neurons is limited. We recently reported quantitative ultrastructural, 
optogenetic, and electrophysiological evidence that VTA glutamate-releasing neurons (VGluT2 ${ }^{+} \mathrm{VGaT}^{-}$) receive a strong glutamatergic input from the lateral hypothalamic area, LHA (Barbano et al., 2020). Furthermore, we also showed that the somatodendritic region of a single VTA glutamate-releasing neuron receives multiple asymmetric synapses from axon terminals arising from LHA glutamatergic neurons (Barbano et al., 2020). Thus, these observations underscore the importance that characterization of the precise synaptic organization between different classes of VTA neuronal types and specific afferents, from neurons of the same nature and shared brain structure, provides on achieving a better understanding on the firing regulation of diverse classes of VTA neurons.

Previous studies found hyperpolarization-induced rebound burst firing in subsets of VTA dopamine and nondopamine neurons mediated by $I_{\mathrm{h}}$ (Tateno and Robinson, 2011) or T-type calcium channels (Tracy et al., 2018, Woodward et al., 2019). We found the same types of responses in subpopulations of glutamate-GABA co-releasing, glutamate-releasing and GABA-releasing neurons. A recent VTA electrophysiological study in a VGaT Knock-in rat line expressing the fluorescent protein Venus reported two populations with different rebound firing properties (type 1 with low threshold calcium spikes during rebound firing, and type two with post hyperpolarizing action potentials during rebound firing) of VGaT-Venus neurons expressing T-channels (Woodward et al., 2019). Given that we detected Tchannel mediated rebound in glutamate-GABA co-releasing and GABA-releasing neurons, and both classes of neurons express VGaT (Root et al., 2018b), it remains to be determined the extent to which these two classes of neurons overlapped with those detected in rat VTA VGaT-Venus neurons.

Findings from electrophysiological and pharmacological studies show presynaptic and postsynaptic MOR function in the VTA (Johnson and North 1992, Margolis et al., 2012; Fields and Margolis, 2015). While postsynaptic MORs are generally thought to be limited to GABA neurons within the VTA, we observed transcripts encoding MORs (Oprm1) expressed in a subset of glutamate-releasing neurons, and these neurons were clustered in the midline aspects of the VTA. We also found that MOR activation hyperpolarizes these glutamate-releasing neurons. These findings of postsynaptic actions of MORs within two subpopulations of VTA neurons together with the presence of MORs in synaptic terminals (Margolis et al., 2004, Zhang et al., 2015, Chen et al., 2015, Bull et al., 2017) underscore the complex actions of opioids within the VTA.

In summary, we detected unique as well as overlapping electrophysiological properties among the glutamate-GABA co-releasing, glutamate-releasing and GABA-releasing VTA neurons. Our electrophysiological findings indicate that firing of VTA glutamate-GABA co-releasing and glutamate-releasing neurons may require stronger excitatory drive compared to the GABA-releasing neurons. However, given that the neuronal firing pattern depends on both the intrinsic properties of the neurons and the network activity innervating them, future studies are necessary to identify the origin, nature, and impact of inputs to the specific classes of VTA neurons. 


\section{Materials and Methods}

\section{Experimental subjects:}

Both male and female mice were used in this study. The vglut2-IRES::Cre mice (JAX \# 016963) and vgat::FlpO mice (JAX \# 031331; Daigle et al., 2018) were crossed to produce a vglut2-IRES::Cre $x$ vgat::FlpO mice. Animals were housed in temperature- and humidity-controlled facilities under a $12 \mathrm{~h}$ light/dark cycle with dawn at $0700 \mathrm{~h}$ and ad libitum chow and water prior to the start of experimental procedures. Mice were 2-3 months of age at the start of the experiment. Experiments were conducted in accordance with the USPHP Guide for the Care and Use of Laboratory Animals and approved by the Animal Care and Use Committee of the National Institute on Drug Abuse Intramural Research Program.

\section{Surgery and Virus Injections:}

Mice were anesthetized with isoflurane (2-4\% induction; $1 \%$ maintenance) and secured to a stereotaxic frame. After exposing the top of the skull, the mouse's head was leveled to ensure the skull was flat. One of the following 3 viruses were injected into the VTA $(0.3 \mu \mathrm{l}$; AP: -3.1 to -3.3 , ML: \pm 0.0 , DV: -4.3 to -4.4$)$ to label the different classes of VTA

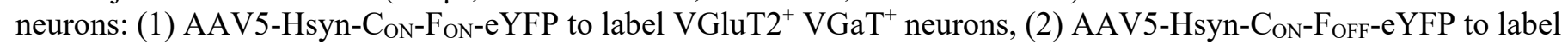
VGluT2 $^{+}$VGaT $^{-}$neurons or (3) AAV5-Hsyn-C OFF-F $_{\text {ON-eYFP to label VGluT2 }}{ }^{-} \mathrm{VGaT}^{+}$neurons. Injections were made using a Micro4 controller and UltraMicroPump along with $10 \mu 1$ Nanofil syringes equipped with 35-gauge needles (WPI Inc., Sarasota, FL). Syringes were left in place for $10 \mathrm{~min}$ following injections to minimize diffusion. Following surgery, mice recovered on a warm heating pad before being transferred back to the vivarium home cage. Mice remained in the colony to allow for recovery and virus expression for 6-8 weeks for RNAscope experiments and 4-6 weeks prior electrophysiology experiments.

\section{Combination of RNAscope in situ Hybridization and Immunolabeling}

Tissue preparation: Wild-type mice and Vglut2-IRES::Cre $x$ Vgat::FlpO mice of six to eight weeks following virus injections were anesthetized with chloral hydrate $(0.5 \mathrm{ml} / \mathrm{kg})$ and perfused transcardially with $4 \%(\mathrm{w} / \mathrm{v})$ paraformaldehyde (PF) in $0.1 \mathrm{M}$ phosphate buffer (PB), pH 7.3. Brains were left in $4 \% \mathrm{PF}$ for $2 \mathrm{~h}$ and transferred to $18 \%$ sucrose in PB overnight at $4{ }^{\circ} \mathrm{C}$ to prepare them for RNAscope in situ hybridization-immunohistochemistry experiments. The detection of transcripts encoding VGluT2 mRNA, VGaT mRNA and Oprm1 mRNA were done by using RNAscope, and TH detection by immunohistochemistry. Coronal free-floating sections (wild-type mouse, VTA, $16 \mu \mathrm{m}$ ) were incubated for 2 $\mathrm{h}$ at $30^{\circ} \mathrm{C}$ with Mouse anti-TH antibody (1:1000, MAB318, Millipore, Burlington, MA) in DEPC-treated phosphate buffer (PB) with $0.5 \%$ Triton X-100 supplemented with RNasin (Promega, Madison, WI). Sections were rinsed $3 \times 10$ min with DEPC-treated PB, and incubated with secondary Donkey anti-Mouse Alexa Fluor 750 (1:100, ab175738, abcam, Cambridge, MA) for $1 \mathrm{~h}$ at $30^{\circ} \mathrm{C}$. Sections were rinsed with DEPC-treated PB and then were mounted onto Fisher SuperFrost slides and dried overnight at $60^{\circ} \mathrm{C}$. RNAscope in situ hybridization processing was performed in accordance to the manufacturer's instructions (Advanced Cell Diagnostics, Newark, CA). Briefly, sections were treated with heat and protease digestion followed by hybridization with a mixture containing target probes to mouse VGluT2 (319171), VGaT (319191-C3) and Oprm1 (489311-C2). Additional sections were hybridized with the bacterial gene DapB as a negative control, which did not exhibit fluorescent labeling. VGluT2 were detected by Atto 550, VGaT were detected by Alexa 488, and Oprm1 were detected by Atto 647. GFP immunolabeling and detection of mRNA for VGluT2 and VGaT were performed as described above. VTA Coronal free-floating sections (VGluT2-Cre $x$ VGaT-FlpO mouse, $16 \mu \mathrm{m}$ in thickness) were processed for immunodetection of Mouse anti-GFP antibody (1:500, 632381, Takara Bio USA, Inc. Mountain View, CA) and incubated with secondary Donkey anti-Mouse Alexa Fluor 488 (1:100, 715-545-151, Jackson ImmunoResearch, West Grove, PA), after processing by RNAscope in situ hybridization, VGluT2 were detected by Atto 550, VGaT were detected by Atto 647. RNAscope in situ hybridization sections were viewed, analyzed, and photographed with an Olympus FV1000 confocal microscope or a Keyence BZ-X800 microscope. Negative control hybridizations showed negligible fluorophore expression. Neurons were counted when the stained cell was at least $5 \mu \mathrm{m}$ in diameter. Pictures were adjusted to match contrast and brightness by using Adobe Photoshop (Adobe Systems). The number of mice ( $\mathrm{n}=3$ /group; 13-16 sections/mouse) analyzed was based on previous studies in our lab using radioactive detection of VGluT2 mRNA from rat VTA neurons.

\section{Patch-clamp recordings:}


Six to eight weeks after virus injection, mice were anesthetized with isoflurane, decapitated, and the brain was quickly removed and placed in ice-cold artificial cerebrospinal fluid (ACSF), saturated with $95 \% \mathrm{O}_{2}$ and $5 \% \mathrm{CO}_{2}$, and modified to contain (in mM): $92 \mathrm{NMDG}, 20$ HEPES, 25 glucose, $30 \mathrm{NaHCO}_{3}, 1.2 \mathrm{NaH}_{2} \mathrm{PO}_{4}, 2.5 \mathrm{KCl}, 5$ sodium ascorbate, 3 sodium pyruvate, 2 thiourea, $10 \mathrm{MgSO}_{4}, 0.5 \mathrm{CaCl}_{2}$ on a VT-1200 vibratome (Leica, Nussloch, Germany), and sectioned through the VTA in horizontal slices $(200 \mu \mathrm{m}$ thick). The slices were placed in a holding chamber filled with the same solution but held at $32^{\circ} \mathrm{C}$. After 10-15 minutes, slices were transferred to a holding chamber containing room temperature ACSF modified to contain (in mM): $92 \mathrm{NaCl}, 20$ HEPES, 25 glucose, $30 \mathrm{NaHCO}_{3}, 1.2 \mathrm{NaH}_{2} \mathrm{PO}_{4}, 2.5 \mathrm{KCl}, 5$ sodium ascorbate, 3 sodium pyruvate, 2 thiourea, $1 \mathrm{MgSO}_{4}, 2 \mathrm{CaCl}_{2}$. For recordings, slices were transferred to a chamber superfused with $32^{\circ} \mathrm{C}$ ACSF containing (in mM): $125 \mathrm{NaCl}, 2.5 \mathrm{KCl}, 1.25 \mathrm{NaH}_{2} \mathrm{PO}_{4}, 1 \mathrm{MgCl}_{2}, 2.4 \mathrm{CaCl}_{2}, 26 \mathrm{NaHCO}_{3}$, and 11 glucose. Electrodes (2-4M $\Omega$ ) were backfilled with an internal solution containing (in $\mathrm{mM}$ ): 120 potassium gluconate, $8.0 \mathrm{NaCl}, 1.0$ $\mathrm{MgCl}_{2}, 10 \mathrm{HEPES}, 2.0 \mathrm{Mg}$-ATP, $0.3 \mathrm{Na}_{2}$-GTP, 10 ditris-phosphocreatine, 0.2 EGTA and $0.2 \%$ biocytin (pH 7.2; 275-290 mOsm). Cells were visualized on an upright microscope using infrared differential interference contrast video microscopy. Whole-cell voltage clamped, and current clamped recordings were made using a MultiClamp 700B amplifier ( $2 \mathrm{kHz}$ low-pass Bessel filter and $10 \mathrm{kHz}$ digitization) with pClamp 10.3 software (Molecular Devices, Sunnyvale, CA). Firing rate was determined before breaking into the cell under cell attached mode for at least 60 seconds of continuous activity. $I_{\mathrm{h}}$ was measured under voltage clamp mode holding at $-60 \mathrm{mV}$ and stepping to $-120,-100$, and $-80 \mathrm{mV}$ for 1000 ms. Membrane potential and AP properties were measured in current clamp I $=0$ within the first 5 minutes after gaining whole cell access. To determine excitability and firing properties, an input/output curve consisting of depolarizing current steps of $500 \mathrm{~ms}$ duration from 0-150 pA were applied and the number of APs fired during each current step were quantified with pClamp 10.1 software. To determine adaptation of AP firing, ISI of the first 10 AP fired after current injection able to evoke the maximum response was analyzed. Neurons with an increase of more than $50 \%$ of the ISI during the first $10 \mathrm{AP}$ was classified as neuron with adaptation. $I_{\mathrm{A}}$ current was measured in voltage clamp using two step protocol. The first step consisted of a $500 \mathrm{~ms}$ hyperpolarizing pre-pulse $(-120 \mathrm{mV}, 500 \mathrm{~ms})$ followed by increasing depolarizing steps from $-100 \mathrm{mV}$ to $30 \mathrm{mV}$ for $1000 \mathrm{~ms}$. The second step was a depolarizing pre-pulse (40 mV) followed by depolarizing steps from $-100 \mathrm{mV}$ to $30 \mathrm{mv} 1000 \mathrm{~ms}$. $I_{\mathrm{A}}$ was obtained by subtracting the currents generated by the second protocol from the currents generated by the first protocol. In the current clamp recordings where $I_{\mathrm{A}}$ was blocked by 4-AP, the membrane potential of the cell was clamped in the membrane potential before the addition of 4-AP and CNQX was applied to prevent an increase AP firing due to an increase in glutamatergic transmission.

\section{Cluster analysis}

Cluster analysis was applied to data from 131 VTA neurons using 21 electrophysiological parameters that include: membrane potential, membrane capacitance, membrane resistance, time constant, tonic firing frequency, Coefficient of variation of tonic firing frequency, Ih current amplitude, rheobase, action potential threshold, gap between resting membrane potential and action potential threshold, AP amplitude, action potential duration, after hyperpolarized potential amplitude, after hyperpolarized potential duration, after hyperpolarized potential peak, latency to fire action potential at rheobase stimulation, number of action potentials fired at rheobase, number of action potentials fired after hyperpolarization, maximum number of action potentials fired after depolarization, current to induce maximum number of action potentials fired, highest firing frequency. Data from each electrophysiological parameter was normalized to its mean value to prevent over representation of a specific parameter during clustering. Cluster analysis was performed in R-studio software using K-means method and Euclidean distance. The result of the clustering was plotted as dendrogram and a hierarchical tree. A principal component analysis was applied to determine the electrophysiological parameters that best separate the clusters and comparisons between clusters were made using one-way ANOVA and Tukey's posthoc test.

\section{Statistical analysis}

Data in the figures are presented as mean \pm SEM, one-way ANOVA or student's t-test were used to compare group of neurons using prism 5.0 software. $\mathrm{P}<0.05$ was required for significance.

Acknowledgments. This work was supported by the Intramural Research Program of the National Institute on Drug Abuse. We thank Drs. David Root and Francois Vautier for setting the colony of Cre/Flp transgenic mice at NIDA/IRP. We also thank Dr. Nirnath Sah (JHU) for advice on cluster analysis. 
Author Contributions. MM and JM-B. conceptualized and initiated the project. JM-B and IC performed electrophysiological studies. JM-B, IC, GEM-S and EM analyzed electrophysiological data. SM, HW, BL performed immunolabeling studies and quantified neurons from RNAscope studies. H-LW and BL performed RNAscope studies. SZ performed confocal studies and data analysis. MM and JM-B and EM prepared the manuscript with contribution from all authors.

Declaration of Interests. The authors declare no competing interests. 


\section{References}

Barbano, M.F., Wang, H-L., Zhang, S., Miranda-Barrientos. J., Estrin, D.J., Figueroa-Gonzalez, A., Liu, B., Barker, D.J., Morales, M. (2020) VTA glutamatergic neurons mediate innate defensive behaviors. Neuron

Berridge, K.C. (2007) The debate over dopamine's role in reward: the case for incentive salience. Psychopharmacology (Berl). 191(3):391-431.

Bromberg-Martin, E.S., Matsumoto, M., Hikosaka, O. (2010) Dopamine in motivational control: rewarding, aversive, and alerting. Neuron. 68(5):815-34. doi: 10.1016/j.neuron.2010.11.022. Review.

Bull, F.A.,Baptista-Hon, D.T., Lambert, J.J., Walwyn, W., Hales, T.J. (2017) Morphine activation of mu opioid receptors causes disinhibition of neurons in the ventral tegmental area mediated by $\beta$-arrestin 2 and c-Src. Sci Rep. 2017; 7: 9969. Published online 2017 Aug 30. doi: 10.1038/s41598-017-10360-8

Carr, D.B., Sesack, S.R. (2010) GABA-containing neurons in the rat ventral tegmental area project to the prefrontal cortex Synapse. 38(2):114-23.

Chen, M., Zhao, Y., Yang, H., Luan, W., Song, J., Cui, D., Dong, Y., Lai, B., Ma, L., Zheng, P. (2015) Morphine disinhibits glutamatergic input to VTA dopamine neurons and promotes dopamine neuron excitation. Elife. 4. doi: 10.7554/eLife.09275.

Chieng, B., Azriel, Y., Mohammadi, S., Christie, M.J. (2011) Distinct cellular properties of identified dopaminergic and GABAergic neurons in the mouse ventral tegmental area. J Physiol. 589(Pt 15):3775-87. doi:

10.1113/jphysiol.2011.210807. Epub 2011 Jun 6.

Fenno, L.E., Mattis, J., Ramakrishnan, C., Hyun, M., Lee, S.Y., He, M., Tucciarone, J., Selimbeyoglu, A., Berndt, A., Grosenick, L., et al. (2014). Targeting cells with single vectors using multiple-feature Boolean logic. Nat Methods 11, 763-772.

Hnasko, T. S., Hjelmstad, G. O., Fields, H. L. \& Edwards, R. H. (2012) Ventral tegmental area glutamate neurons: electrophysiological properties and projections. J. Neurosci. 32, 15076-15085.

Johnson, S.W., North, R.A. (1992) Opioids excite dopamine neurons by hyperpolarization of local interneurons. J Neurosci 12: 483-488.

Jones, S., Kauer, J.A. (1999) Amphetamine depresses excitatory synaptic transmission via serotonin receptors in the ventral tegmental area. J Neurosci 19:9780-9787.

Khaliq, Z.M., Bean, B.P. (2010) Pacemaking in Dopaminergic Ventral Tegmental Area Neurons: Depolarizing Drive from Background and Voltage-Dependent Sodium Conductances J Neurosci. 30(21): 7401-

7413. doi: 10.1523/JNEUROSCI.0143-10.2010.

Kudo, T., Uchigashima, M., Miyazaki, T., Konno, K., Yamasaki, M., Yanagawa, Y., Minami, M., Watanabe, M. (2012) Three types of neurochemical projection from the bed nucleus of the stria terminalis to the ventral tegmental area in adult mice. J. Neurosci. 32(50):18035-46. doi: 10.1523/JNEUROSCI.4057-12.2012.

Kudo T, Konno K, Uchigashima M, Yanagawa Y, Sora I, Minami M, Watanabe M. (2014) GABAergic neurons in the ventral tegmental area receive dual GABA/enkephalin-mediated inhibitory inputs from the bed nucleus of the stria terminalis. Eur J Neurosci. 39(11):1796-809. doi: 10.1111/ejn.12503. 
Li, X., Qi, J., Yamaguchi, T., Wang, H.L., Morales, M. (2013) Heterogeneous composition of dopamine neurons of the rat A10 region: molecular evidence for diverse signaling properties. Brain Struct Funct. 218(5):1159-76. doi: 10.1007/s00429-012-0452-z. Epub 2012 Aug 29.

Margolis, E.B., Hjelmstad, G.O., Bonci, A., Fields, H.L. (2005). Both kappa and mu opioid agonists inhibit glutamatergic input to ventral tegmental area neurons. Journal of Neurophysiology 93:3086-3093. doi: 10.1152/jn.00855.2004.

Margolis, E. B., Lock, H., Hjelmstad, G. O. \& Fields, H. L. (2006) The ventral tegmental area revisited: is there an electrophysiological marker for dopaminergic neurons? J. Physiol. 577, 907-924.

Margolis, E. B., Toy, B., Himmels, P., Morales, M. \& Fields, H. L. (2012) Identification of rat ventral tegmental area GABAergic neurons. PLoS ONE 7, e42365.

Monteggia. L.M., Eisch, A.J., Tang, M.D., Kaczmarek, L.K., Nestler, E.J. (2000) Cloning and localization of the hyperpolarization-activated cyclic nucleotide-gated channel family in rat brain._Brain Res Mol Brain Res. 81(12):129-39.

Morales, M., Margolis, E.B. (2017) Ventral tegmental area: cellular heterogeneity, connectivity and behaviour. Nat Rev Neurosci. 18(2):73-85. doi: 10.1038/nrn.2016.165. Epub 2017 Jan 5. Review

Nieh, E.H., Matthews, G.A., Allsop, S.A., Presbrey, K.N., Leppla, C.A., Wichmann, R., Neve, R., Wildes, C.P., Tye, K.M. (2015) Decoding neural circuits that control compulsive sucrose seeking. Cell. 160(3):528-41. doi: 10.1016/j.cell.2015.01.003.

Notomi, T., Shigemoto, R. (2004) Immunohistochemical localization of Ih channel subunits, HCN1-4, in the rat brain. J Comp Neurol. 471(3):241-76.

Ntamati, Niels R., Creed, Meaghan, Achargui Ridouane, Lüscher Christian (2018) Periaqueductal efferents to dopamine and GABA neurons of the VTA PLoS One. 13(1): e0190297. Published online 2018 Jan 5. doi: 10.1371/journal.pone.0190297

Omelchenko, N., Bell, R., Sesack, SR. (2009) Lateral habenula projections to dopamine and GABA neurons in the rat ventral tegmental area. Eur J Neurosci. 30(7):1239-50. doi: 10.1111/j.1460-9568.2009.06924.x. Epub 2009 Sep 29

Omelchenko, N., Sesack, SR. (2010) Periaqueductal gray afferents synapse onto dopamine and GABA neurons in the rat ventral tegmental area J Neurosci Res. 88(5):981-91. doi: 10.1002/jnr.22265..

PMID:

Root, D.H., Estrin, D.J., Morales, M. (2018b) Aversion or Salience Signaling by Ventral Tegmental Area Glutamate Neurons. iScience. 2:51-62. doi: 10.1016/j.isci.2018.03.008.

Root, D.H., Mejias-Aponte, C.A., Zhang, S., Wang, H.L., Hoffman, A.F., Lupica, C.R., and Morales, M. (2014). Single rodent mesohabenular axons release glutamate and GABA. Nat Neurosci 17, 1543-1551.

Root, D.H., Zhang, S., Barker, D.J., Miranda-Barrientos, J., Liu, B., Wang, H.L., and Morales, M. (2018). Selective Brain Distribution and Distinctive Synaptic Architecture of Dual Glutamatergic-GABAergic Neurons. Cell Rep 23, 3465-3479

Tan, K.R., Yvon, C., Turiault, M., Mirzabekov, J.J., Doehner, J., Labouèbe, G., Deisseroth, K., Tye, K.M., Lüscher (2012) GABA neurons of the VTA drive conditioned place aversion. Neuron 73, 1173-1183. Tateno, T. Robinson, H.P. (2011) The mechanism of ethanol action on midbrain dopaminergic neuron firing: a dynamic-clamp study of the role of I(h) and GABAergic synaptic integration. J Neurophysiol. 106(4):1901-22. doi: 10.1152/jn.00162.2011. Epub 2011 Jun 22 
Tracy, M.E., Tesic, V., Stamenic, T.T., Joksimovic, S.M., Busquet, N., Jevtovic-Todorovic, V., Todorovic, S.M. (2018) Cav3.1 isoform of T-type calcium channels supports excitability of rat and mouse ventral tegmental area neurons. Neuropharmacology. 135:343-354. doi: 10.1016/j.neuropharm.2018.03.028. Epub 2018 Mar 23.

van Zessen, R., Phillips, J. L., Budygin, E. A. \& Stuber, G. D. (2012) Activation of VTA GABA neurons disrupts reward consumption. Neuron 73, 1184-1194.

Wise RA (2004) Dopamine, learning and motivation. Nat Rev Neurosci. 2004;5:483-494.

Woodward, T.J., Tesic, V., Stamenic, T.T., Jevtovic-Todorovic, V., Todorovic, S.M. (2019) Pharmacological Antagonism of T-Type Calcium Channels Constrains Rebound Burst Firing in Two Distinct Subpopulations of GABA Neurons in the Rat Ventral Tegmental Area: Implications for $\alpha$-Lipoic Acid. Front Pharmacol. 10:1402. doi: 10.3389/fphar.2019.01402. eCollection 2019.

Yamaguchi, T., Sheen, W., Morales, M. (2007) Glutamatergic neurons are present in the rat ventral tegmental area. Eur J Neurosci 25:106-118.

Zhang, W., Yang, H.L., Song, J.J., Chen, M., Dong, Y., Lai, B., Yu, Y.G., Ma, L., Zheng, P. (2015) DAMGO depresses inhibitory synaptic transmission via different downstream pathways of $\mu$ opioid receptors in ventral tegmental area and periaqueductal gray. Neuroscience. 301:144-54. doi: 10.1016/j.neuroscience.2015.05.077. Epub 2015 Jun 3.

Zhou, Z., Liu, X., Chen, S., Zhang, Z., Liu, Y., Montardy, Q., Tang, Y., Wei, P., Liu, N., Li, L., Song, R., Lai, J., He, X., Chen, C., Bi, G., Feng, G., Xu, F., Wang, L. (2019) A VTA GABAergic Neural Circuit Mediates Visually Evoked Innate Defensive Responses. Neuron. 103(3):473-488.e6. doi: 10.1016/j.neuron.2019.05.027. Epub 2019 Jun 12. 

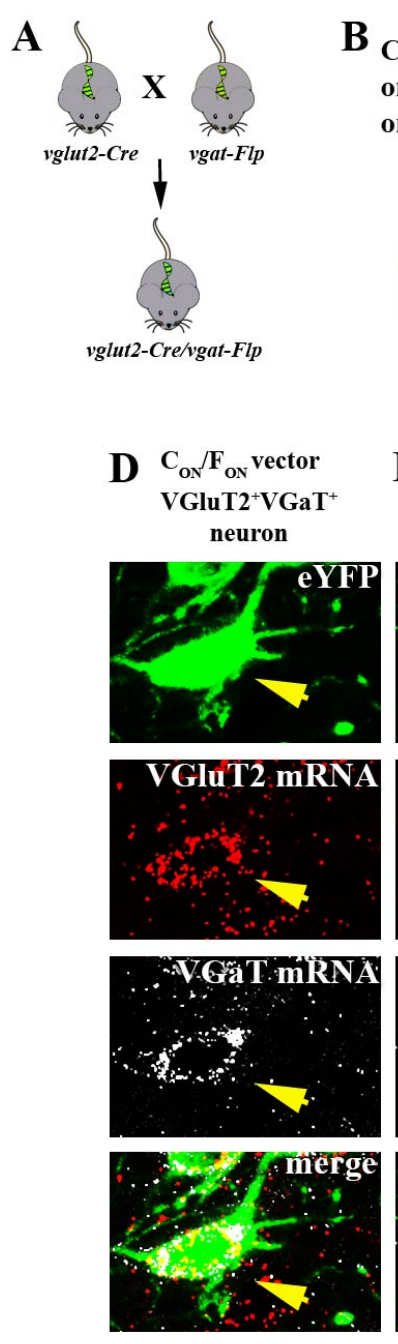

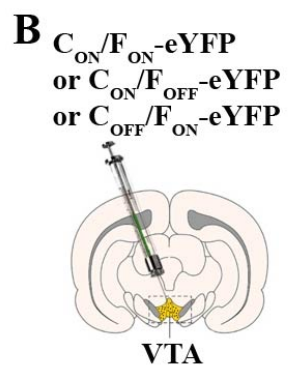

VTA
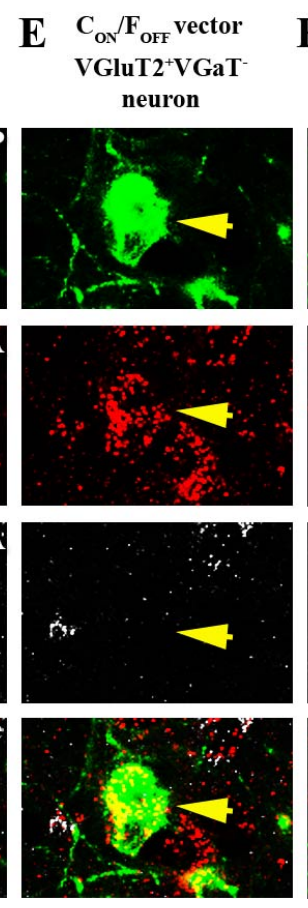
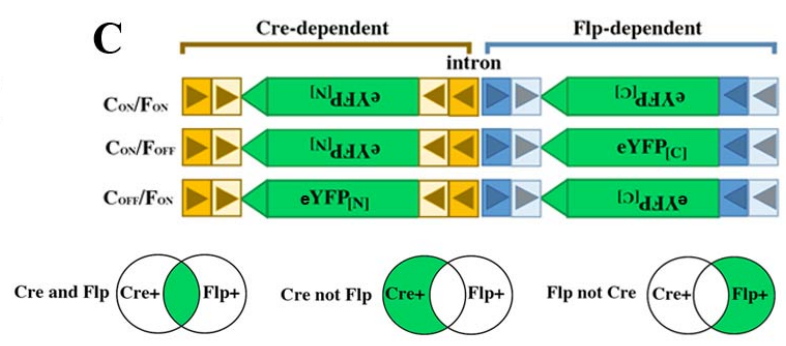

G VTA transfected neurons expressing F $\quad \begin{gathered}\mathrm{C}_{\text {ofF }} / \mathrm{F}_{\text {ov }} \text { vector } \\ \text { VGluT2 } 2 \text { VGaT }^{+}\end{gathered}$ neuron
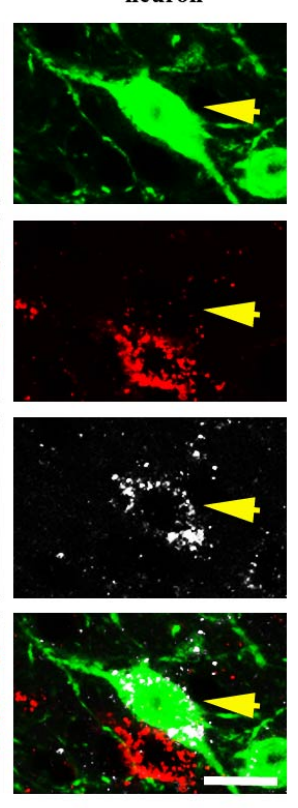
VGluT2 or VGaT mRNAs

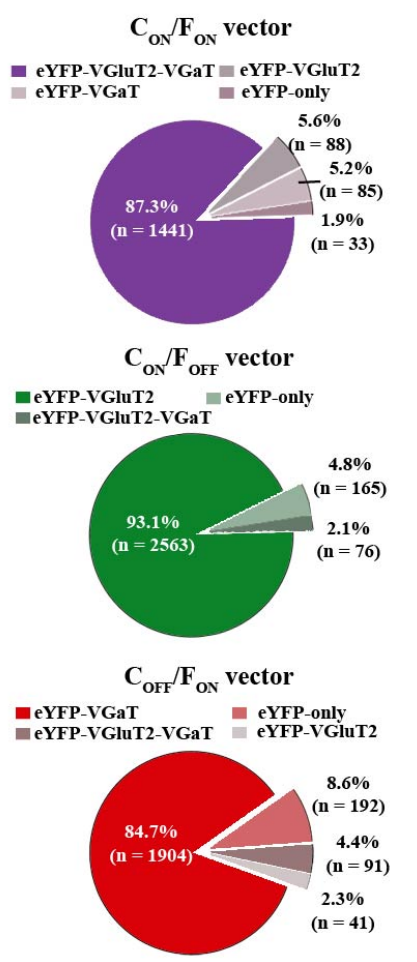

Figure 1. Selective targeting of VTA VGluT2 ${ }^{+} \mathrm{VGaT}^{+}$, VGluT2 ${ }^{+} \mathrm{VGaT}^{-}$and VGluT2- $\mathrm{VGaT}^{+}$neurons. (AC) Crossing of vglut2-Cre and vgat-Flp mice to generate vglut2-Cre/vgat-Flp mouse and intra-VTA injections of INTRSECT AAV-Con/Fon-eYFP to target VGluT2 ${ }^{+} \mathrm{VGaT}^{+}$neurons, AAV-Con/FofF-eYFP to target $\mathrm{VGluT2}^{+} \mathrm{VGaT}^{-}$neurons or AAV-CofF/Fon-eYFP vectors to target VGluT2 ${ }^{-} \mathrm{VGaT}^{+}$neurons. (D) Coexpression of VGluT2 mRNA and VGaT mRNA in VTA VGluT2 ${ }^{+} \mathrm{VGaT}^{+}$eYFP neuron. (E) VGluT2 mRNA without VGaT mRNA in VTA VGluT2 ${ }^{+}$VGaT $^{-}$eYFP neuron. (F) VGaT mRNA without VGluT2 mRNA in VTA VGluT2 ${ }^{-} \mathrm{VGaT}^{+}$eYFP neuron. Scale bar $=20 \mathrm{uM}(\mathbf{G})$ Total percentage of VTA transfected neurons co-expressing VGluT2 mRNA or VGaT mRNA ( $\mathrm{n}=3$ mice per group). 
A

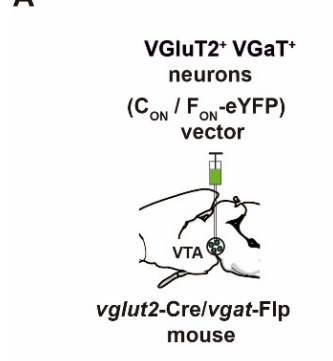

B

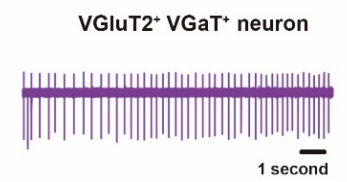

C

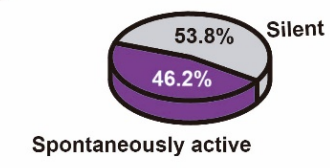

D

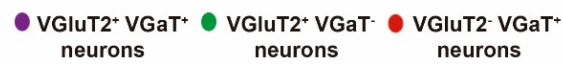

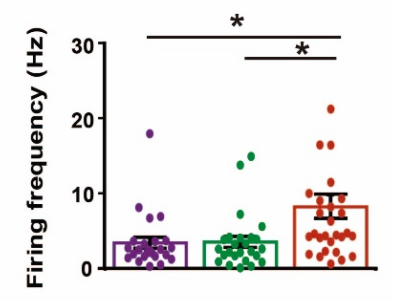

F
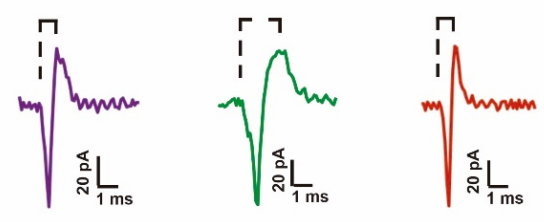
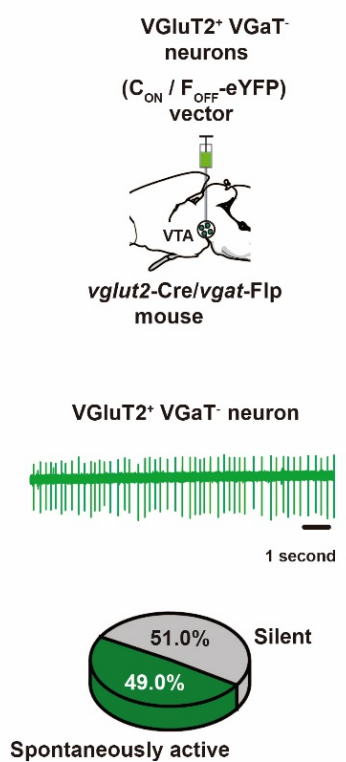

E
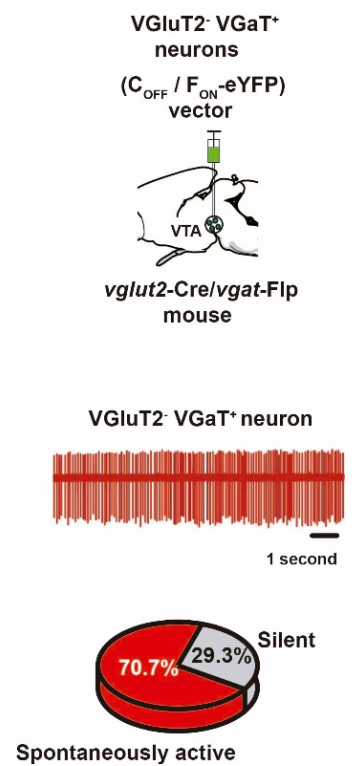

Spontaneously active

Figure 2. Spontaneous firing activity in VTA VGluT2 ${ }^{+} \mathrm{VGaT}^{+}, \mathrm{VGluT2}^{+} \mathrm{VGaT}^{-}$, and VGluT2- $\mathrm{VGaT}^{+}$ neurons during ex vivo cell attached recordings. (A) Schematic representation of intra-VTA injections of vectors (AAV-Con/Fon- eYFP, AAV-Con/FofF-eYFP and AAV-CofF/Fon- eYFP) in vglut2-Cre/vgat-Flp mice. (B) Traces recorded in the cell attached configuration in horizontal slices from identified spontaneously active VTA neurons. (C) Proportion of VTA spontaneously active vs quiescent neurons. (D) Summary of spontaneous firing rate across VTA neurons. VGluT2- $\mathrm{VGaT}^{+}$neurons have higher firing frequencies than VGluT2 $^{+} \mathrm{VGaT}^{+}$and VGluT2 ${ }^{+}$VGaT $^{-}$neurons. One-way ANOVA F $2,77=5.795 \mathrm{p}=0.046$; Tukey's post hoc test ${ }^{*} \mathrm{p}<0.05$. (E) There is no relationship between firing frequency and coefficient of variation $(\mathrm{CV})$ of interspike intervals (ISIs) for these neuronal types. Inset, summary of CV of ISIs in VGluT2 ${ }^{+} \mathrm{VGaT}^{+}$, VGluT2 ${ }^{+}$ $\mathrm{VGaT}^{-}$, and VGluT2- ${ }^{-} \mathrm{VGaT}^{+}$neurons. Example traces $(\mathbf{F})$ and summary durations $(\mathbf{G})$ of extracellular recorded APs. One-way ANOVA $\mathrm{F}_{2,77}=6.745=0.0026$; Tukey's post hoc test $* \mathrm{p}<0.05, * * \mathrm{p}<0.01$ 
A
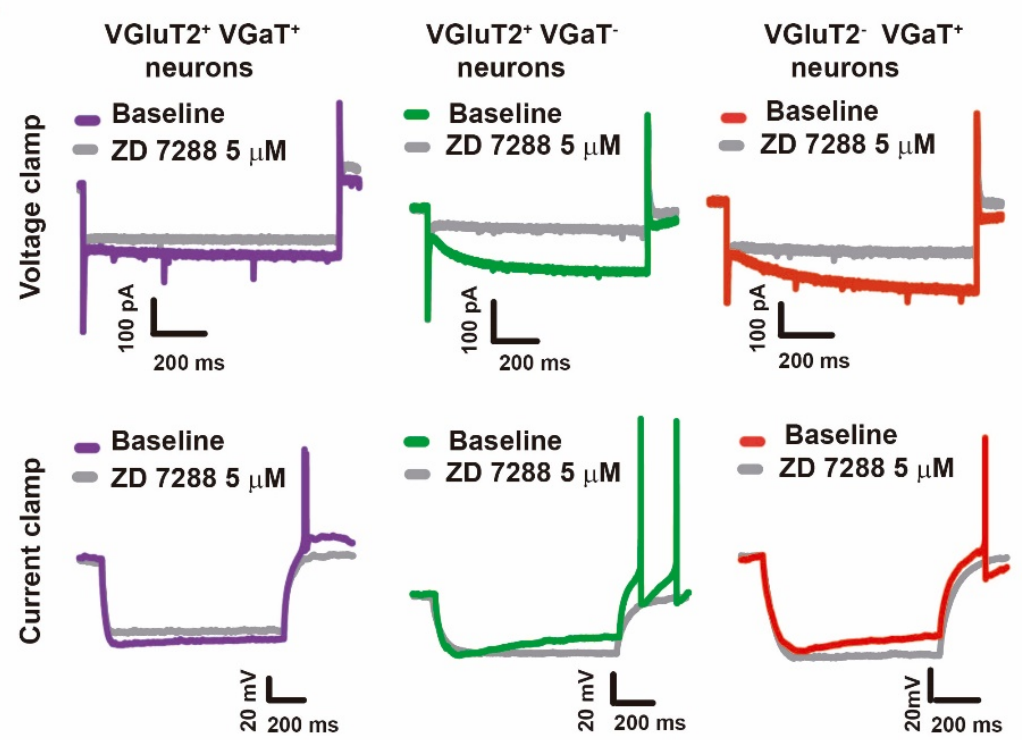

B

C

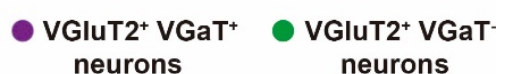

- VGluT2- $\mathrm{VGaT}^{+}$ neurons
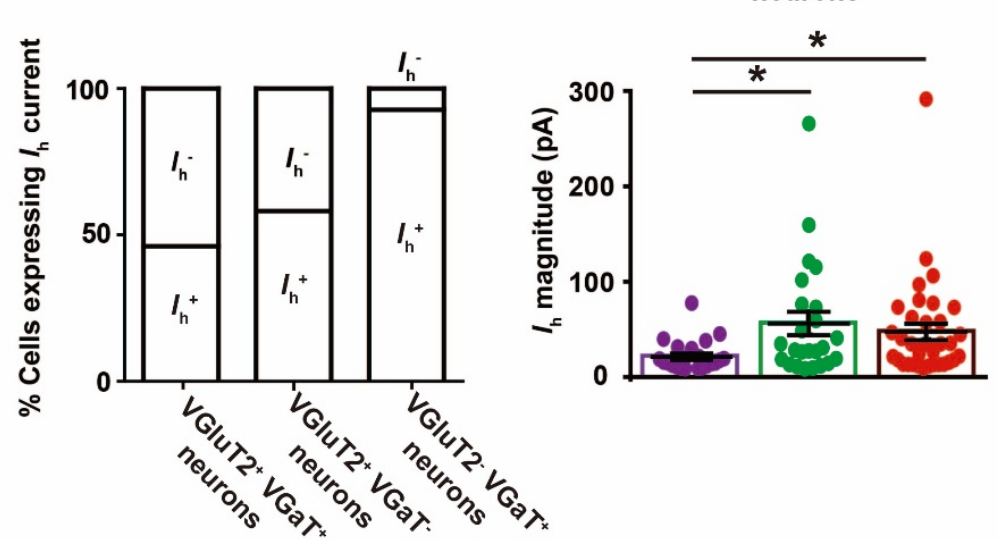

Figure 3. Many VTA VGluT2 ${ }^{+}$VGaT $^{+}$, VGluT2 ${ }^{+}$VGaT $^{-}$, and VGluT2 ${ }^{-}$VGaT $^{+}$neurons have an $I_{h}$. (A) Voltage clamp (top; step from $-60 \mathrm{mV}$ to $-120 \mathrm{mV}$, 1s duration) and current clamp (bottom; step from 0 pA to $50-100 \mathrm{pA}$ to reach $\mathrm{mV}=-120 \mathrm{mV}$ ) traces of $I_{\mathrm{h}}$ measurements in VTA neurons. $\boldsymbol{I}_{\mathbf{h}}$ was blocked by ZD 7288 (5 $\mu \mathrm{M}$; gray traces). (B) Proportion of each VTA neuronal types that expressed $I_{\mathrm{h}}$. (C) Smaller $I_{\mathrm{h}}$ amplitude was observed in VGluT2 ${ }^{+} \mathrm{VGaT}^{+}$neurons compared to $\mathrm{VGluT}^{+} \mathrm{VGaT}^{-}$or $\mathrm{VGluT}^{-} \mathrm{VGaT}^{+}$neurons. One-way ANOVA $F_{2,82}=3.528 p=0.034$, Tukey's post hoc test $* \mathrm{p}<0.05$. 
A

D
B

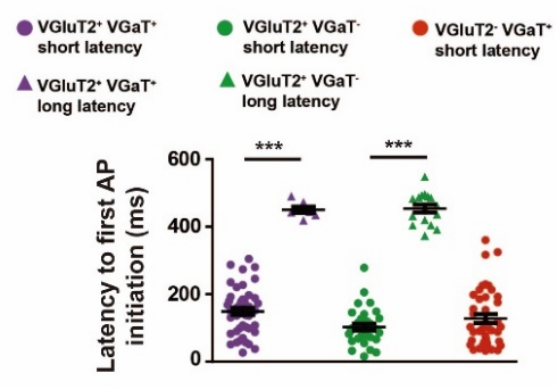

C

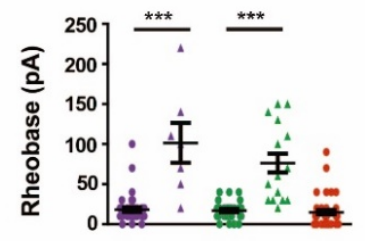

VGluT2+ VGaT+ neurons Long latency $(\mathrm{N}=7)$

Short Latency $(\mathrm{N}=41)$

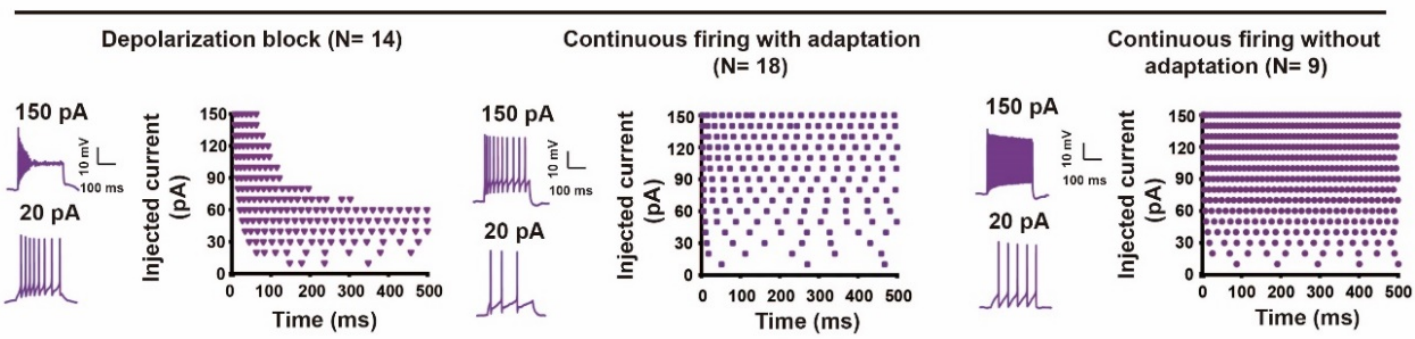

VGluT2+ VGaT' neurons

Long latency $(\mathrm{N}=16)$

Short Latency $(\mathrm{N}=29)$

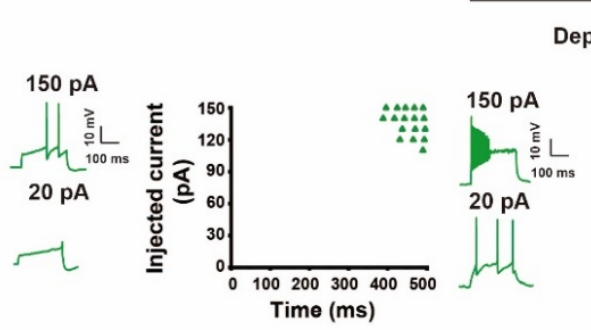

Deporization block ( $\mathrm{N}=5$ )

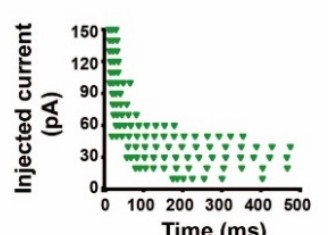

Continuous firing with adaptation $(\mathrm{N}=10)$
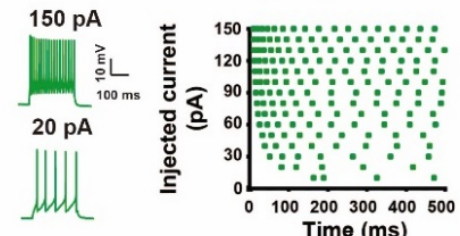

Time (ms)

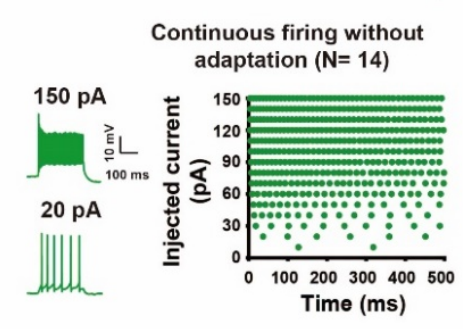

VGluT2 VGaT $^{+}$neurons

Short Latency $(\mathrm{N}=\mathbf{3 8})$

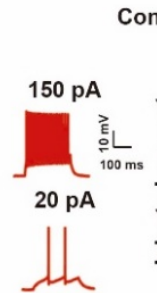

Continuous firing with adaptation $(\mathrm{N}=16)$

Continuous firing without adaptation $(\mathrm{N}=22)$

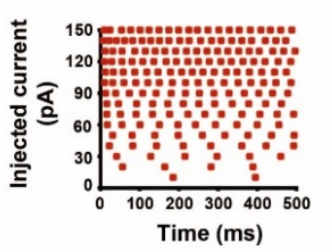

Figure 4. Stimulated firing patterns of VTA VGluT2 ${ }^{+} \mathrm{VGaT}^{+}, \mathrm{VGluT2}^{+} \mathrm{VGaT}^{-}$, and $\mathrm{VGluT2}^{-} \mathrm{VGaT}^{+}$ neurons. (A) Example current clamp traces of VTA neurons with long or short latency AP firing in response to injected current steps. (B) VGluT2 ${ }^{+} \mathrm{VGaT}^{+}$, $\mathrm{VGluT}^{+} \mathrm{VGaT}^{-}$, and $\mathrm{VGluT}^{-} \mathrm{VGaT}^{+}$neurons with short or long latency to first AP. One-way ANOVA $\mathrm{F}_{4,139}=107.8 \mathrm{p}<0.0001$, Tukey's post hoc test ***p $<0.0001$ (C) Evoked AP firing in $\mathrm{VGluT}^{+} \mathrm{VGaT}^{+}$, VGluT2 ${ }^{+} \mathrm{VGaT}^{-}$, and $\mathrm{VGluT2}^{-} \mathrm{VGaT}^{+}$neurons. Neurons with long latency to firing mostly required current injections $>40 \mathrm{pA}$ to drive firing. One-way ANOVA $\mathrm{F}_{4,13}=32.49 \mathrm{p}<0.0001$, Tukey's post hoc test $* * * \mathrm{p}<0.0001$. (D) Example firing patterns in response to injected current steps sorted by long or short latency to firing. Short latency firing VGluT2 ${ }^{+} \mathrm{VGaT}^{+}$and $\mathrm{VGluT2}^{+} \mathrm{VGaT}^{-}$neurons go into depolarization block, show continuous firing with adaptation, or show continuous firing without adaptation. VGluT2- $\mathrm{VGaT}^{+}$neurons only show responses of continuous firing with or without adaptation. 
A

$\square$ VGluT2+ VGaT $^{+}$neurons with continuous firing with adaptation

- VGluT2+ VGaT+ neurons with continuous firing without adaptation

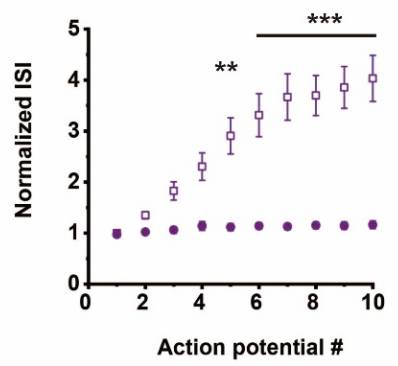

B

$\square$ VGluT2+ VGaT neurons with continuous firing with adaptation

- VGluT2+ VGaT neurons with continuous firing without adaptation

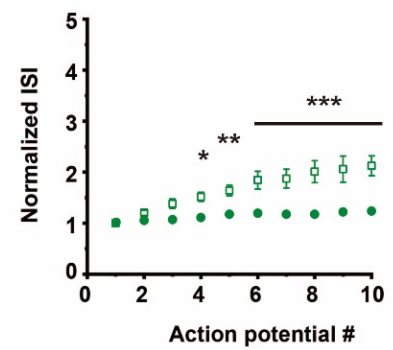

C

VGluT2- VGaT ${ }^{+}$neurons with continuous firing with adaptation

- VGluT2- VGaT ${ }^{+}$neurons with continuous firing without adaptation

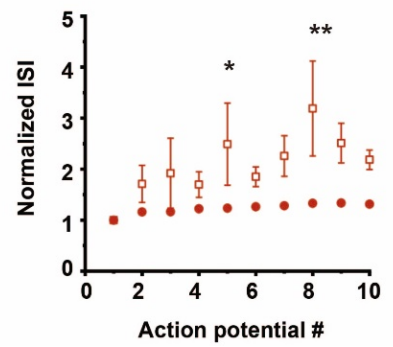

Figure 4-figure supplement 1 . Neurons that fire with adaptation during depolarizing current steps have larger inter-spike intervals (ISIs) than neurons without adaptation. (A-C) Normalized ISI of the first 10 AP fired after $150 \mathrm{pA}$ current injection from (A) VGluT2 ${ }^{+} \mathrm{VGaT}^{+}$neurons with adaptation and without adaptation. Two way ANOVA spike number $x$ type of neuorn $\mathrm{F}_{9,250}=4.72 \mathrm{p}<0.0001$, type of neuron $\mathrm{F}_{1,250}=$ $121.44 \mathrm{p}<0.0001$, spike number $\mathrm{F}_{9,250}=5.73 \mathrm{p}<0.0001$ Bonferroni posthoc test $* * \mathrm{p}<0.01,{ }^{* * *} \mathrm{p}<0.001$. (B) VGluT2 $^{+}$VGaT $^{-}$neurons with adaptation and without adaptation Two way ANOVA spike number $\mathrm{x}$ type of neuorn $\mathrm{F}_{9,220}=5.08 \mathrm{p}<0.0001$, type of neuron $\mathrm{F}_{1,220}=147.51 \mathrm{p}<0.0001$, spike number $\mathrm{F}_{9,220}=11.31 \mathrm{p}<0.001$. Bonferroni posthoc test $* \mathrm{p}=0.05, * * \mathrm{p}<0.01, * * * \mathrm{p}<0.001$ and $(\mathbf{C})$ VGluT2 ${ }^{-} \mathrm{VGaT}^{+}$neurons with adaptation and without adaptation Two way ANOVA spike number $x$ type of neuorn $F_{9,360}=1.39 \mathrm{p}=0.1904$, type of neuron $\mathrm{F}_{1,360}=38.80 \mathrm{p}<0.0001$, spike number $\mathrm{F}_{9,360}=2.48 \mathrm{p}<0.094$. Bonferroni posthoc test ${ }^{*} \mathrm{p}=0.05,{ }^{*} \mathrm{p}<$ 0.01 . 
A

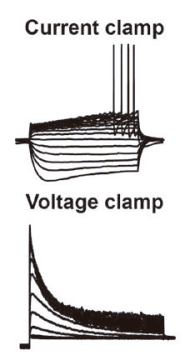

C

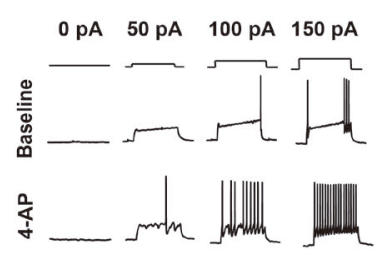

D

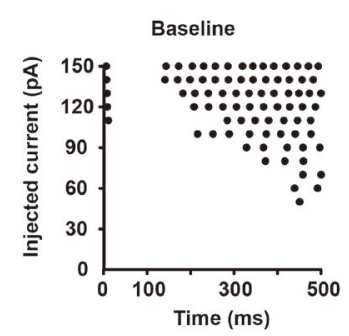

$\mathbf{F}$

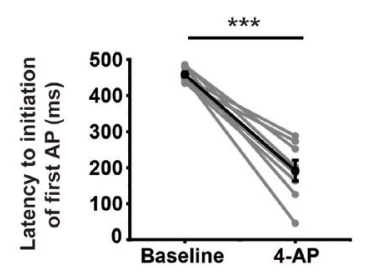

B

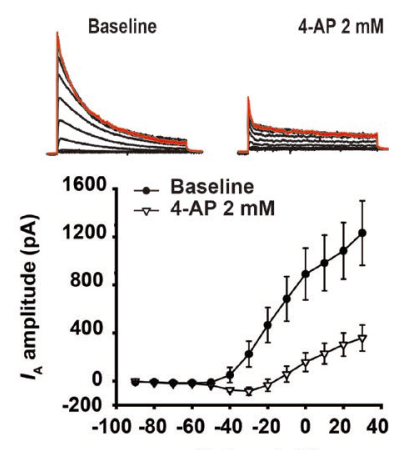

Voltage $(\mathrm{mV})$

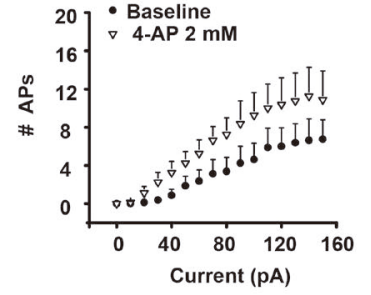

E

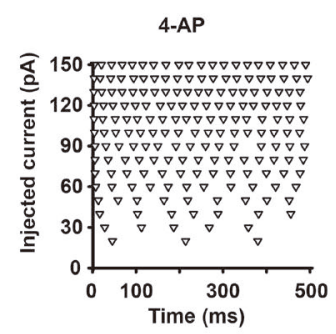

G

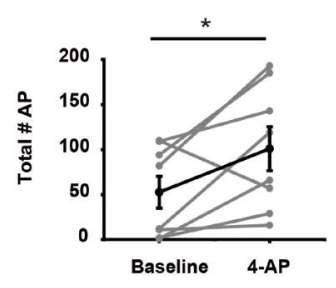

Figure 4-figure supplement 2. $I_{\mathrm{A}}$ mediates long latency AP firing. (A) Example current clamp (top) and voltage clamp (bottom) traces in a VGluT2 ${ }^{+} \mathrm{VGaT}^{-}$long latency neuron (B) Example voltage clamp traces from a VGluT2 ${ }^{+} \mathrm{VGaT}^{-}$long latency neuron at baseline and after bath application of the $I_{\mathrm{A}}$ blocker 4Aminopyridine (4-AP) (top). Mean $I_{\mathrm{A}}$ amplitude responses across different voltage steps at baseline (close circles) and after bath application of 4-AP (open triangles) (bottom) $\left(\mathrm{n}=12\right.$ neurons, 3 VGluT2 ${ }^{+} \mathrm{VGaT}^{+}$ neurons and $9 \mathrm{VGluT2}^{+} \mathrm{VGaT}^{-}$) (C) Example current clamp traces (left) of a long latency VGluT2 ${ }^{+} \mathrm{VGaT}^{+}$ neuron during depolarizing step current injections before and after bath application of 4-AP. Input/output curves show 4-AP (open triangles) increased number of APs fired during depolarizing current injections compared to baseline (closed circles) ( $\mathrm{n}=8$ neurons, $4 \mathrm{VGluT}^{+} \mathrm{VGaT}^{+}$neurons and $4 \mathrm{VGluT2}^{+} \mathrm{VGaT}^{-}$neurons) Two way ANOVA 4-AP x current $\mathrm{F}_{15,112}=0.83 \mathrm{p}=0.6408$, 4-AP $\mathrm{F}_{15,112}=49.06 \mathrm{p}<0.0001$, current $\mathrm{F}_{15,112=4.64 \mathrm{p}<}$ 0.0001. (D-E) Example firing pattern during injected current steps in a $\mathrm{VGluT2}^{+} \mathrm{VGaT}^{+}$long latency neuron at baseline (D) and after bath application of 4-AP (E). (F) 4-AP decreased the latency to AP firing during the minimum injected current step in long latency neurons ( $\mathrm{n}=8$ neurons, $4 \mathrm{VGluT}^{+} \mathrm{VGaT}^{+}$and $4 \mathrm{VGluT}^{+}$ $\mathrm{VGaT}^{-}$). Paired $t$-test $t_{7}=8.877 * * * \mathrm{p}<0.0001$ (G) 4-AP increased total number of APs fired after activation across long latency neurons during an input/output curve $(10-150 \mathrm{pA}, 500 \mathrm{~ms})$. Paired $t$-test $t_{7}=2.426 * \mathrm{p}=$ $0.0457\left(\mathrm{n}=8\right.$ neurons $4 \mathrm{VGluT2}^{+} \mathrm{VGaT}^{+}$and $\left.4 \mathrm{VGluT2}^{+} \mathrm{VGaT}^{-}\right)$ 
A
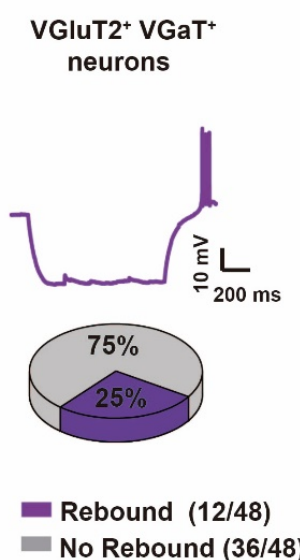

No Rebound (36/48)

B

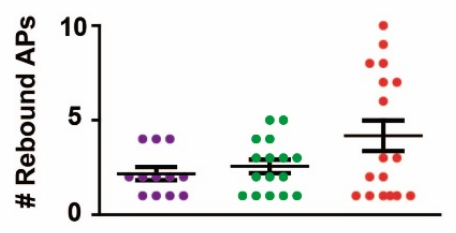

C
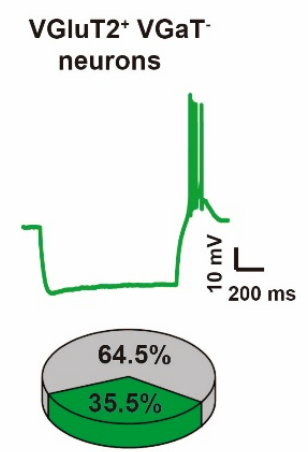

Rebound (16/45)

- No Rebound (29/45)

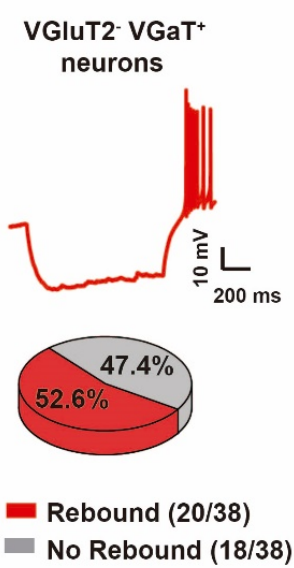

VGluT2- VGaT+

No Rebound (18/38)

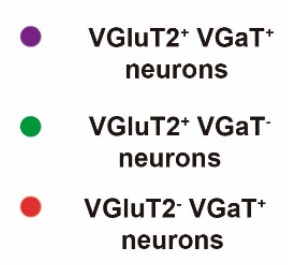
VGluT2 $^{+}$VGaT $^{+}$
neurons
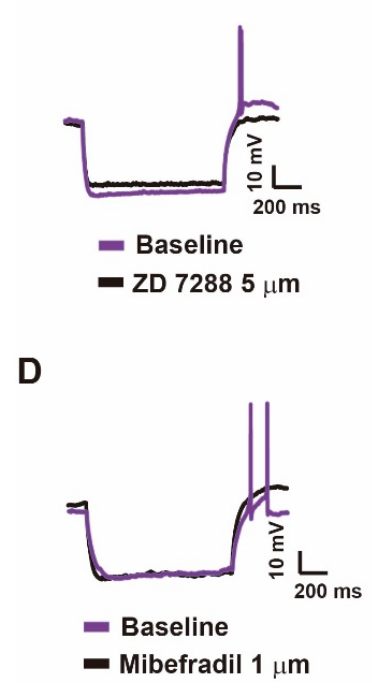
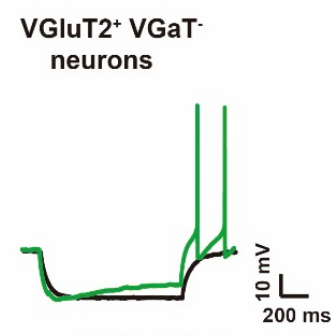

- Baseline

- ZD $72885 \mu \mathrm{m}$

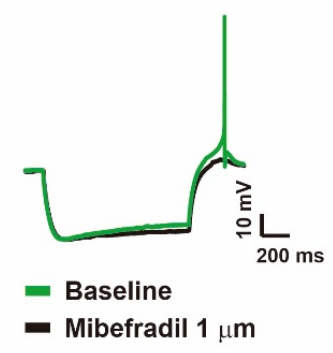

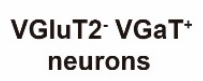
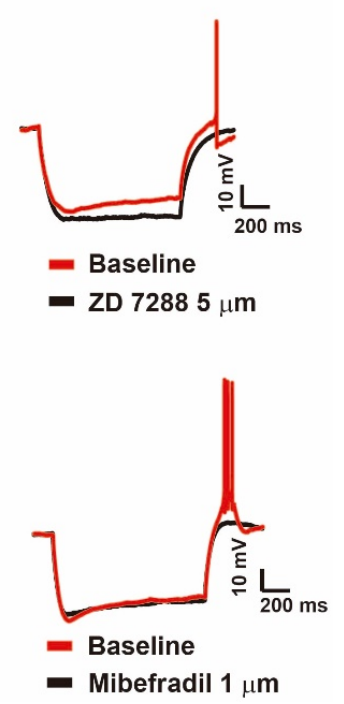

Figure 5. Rebound firing neurons is mediated by $I_{\mathrm{h}}$ and T-type calcium channels in $\mathrm{VGluT2}^{+} \mathrm{VGaT}^{+}$, VGluT2 $^{+}$VGaT $^{-}$, and VGluT2- VGaT $^{+}$neurons. (A) Example current clamp traces (top) and proportion of neurons showing rebound firing (bottom) among $\mathrm{VGluT}^{+} \mathrm{VGaT}^{+}$(purple), $\mathrm{VGluT}^{+} \mathrm{VGaT}^{-}$(green), and VGluT2$^{-} \mathrm{VGaT}^{+}$(red) neurons. (B) Number of rebound APs fired by VGluT2 ${ }^{+} \mathrm{VGaT}^{+}$(purple), $\mathrm{VGluT2}^{+}$ $\mathrm{VGaT}^{-}$(green), and $\mathrm{VGluT}^{-} \mathrm{VGaT}^{+}$(red) neurons. (C) Example recordings in which rebound firing was blocked by ZD 7288 (5uM) (black traces). (D). Example recordings in which rebound firing was blocked by Mibefradil (1uM) (black traces). 

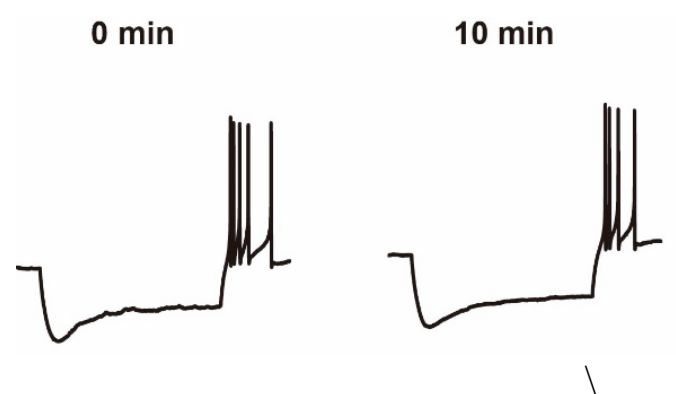

Figure 5-figure supplement 1. Rebound firing is stable across time. (A) Current clamp traces from a VGluT2- $\mathrm{VGaT}^{+}$neuron with rebound firing at the beginning of the experiment ( 0 minutes) and after 10 minutes. (B) Number of rebound APs in at the beginning of the experiment ( 0 minutes) and after 10 minutes of recording ( $\mathrm{n}=8$ neurons, $2 \mathrm{VGluT}^{+} \mathrm{VGaT}^{+}$neurons, $2 \mathrm{VGluT}^{+} \mathrm{VGaT}^{-}$neurons, and $4 \mathrm{VGluT}^{-} \mathrm{VGaT}^{+}$ neurons) 
A

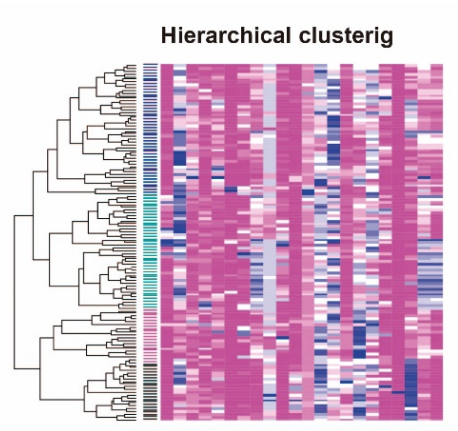

C
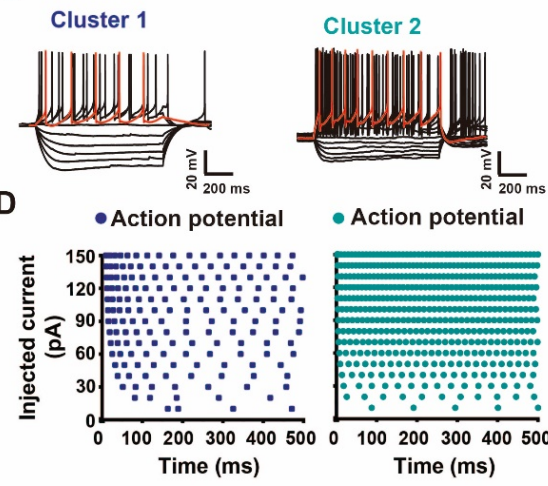

E

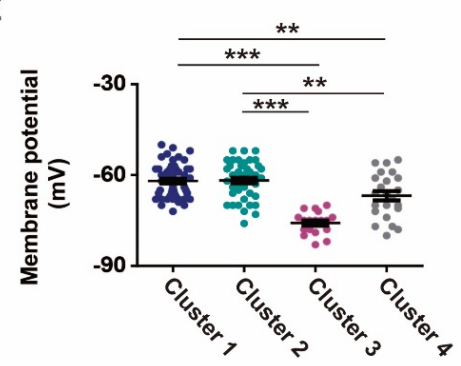

G

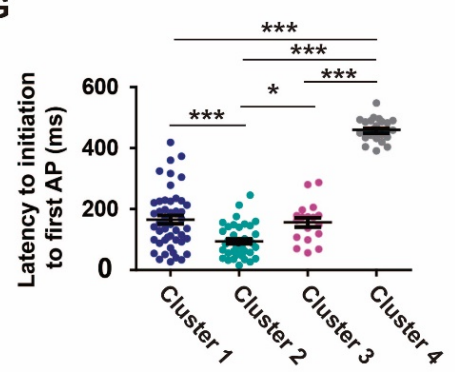

$\mathbf{F}$

H
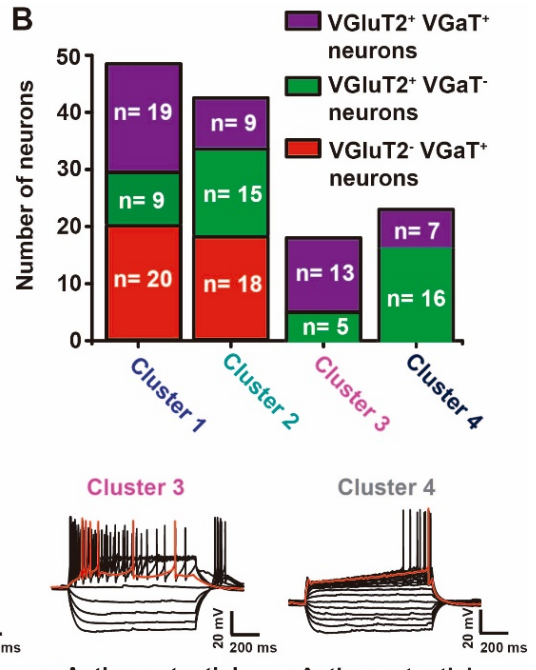

- Action potential Action potential
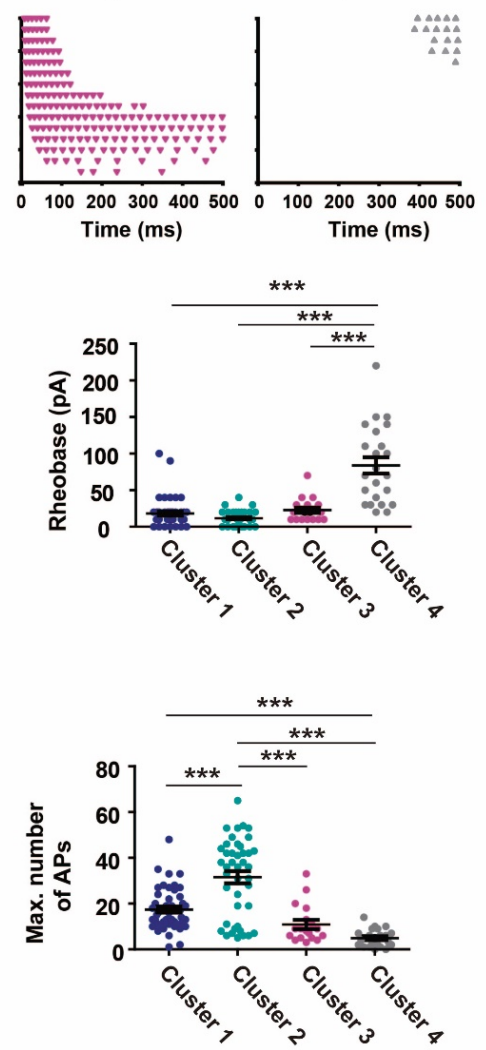

Figure 6. Hierarchical cluster analysis of electrophysiological properties of VTA VGluT2 ${ }^{+} \mathrm{VGaT}^{+}$, VGluT2 $^{+}$VGaT $^{-}$, and VGluT2- VGaT $^{+}$neurons. (A) Dendrogram and heat map of VTA neuronal electrophysiological properties. (B) Neurotransmitter neuronal phenotype distributions across clusters. (C) Example current clamp traces from neurons within each cluster in response to hyperpolarizing and depolarizing current injections. (D) Examples of firing patterns over time during depolarization steps in neurons within each cluster. (E) Membrane potentials of neurons in each cluster. One-way ANOVA $F_{3,130}=28.25 \mathrm{p}<0.0001$, Tukey's post hoc test $* * \mathrm{p}<0.001 * * * \mathrm{p}<0.0001$. (F) Rheobase of neurons in each cluster. One-way ANOVA $\mathrm{F}_{3,130}=$ $42.45 \mathrm{p}<0.0001$, Tukey's post hoc test ***p $<0.0001$. (G) Latency to fire APs in response to depolarizing current steps in neurons in each cluster. One-way ANOVA $\mathrm{F}_{3,130}=134.9 \mathrm{p}<0.0001$, Tukey's post hoc test * $\mathrm{p}<$ $0.05 * * * \mathrm{p}<0.0001$. (H) Maximum number of APs fired during depolarizing current steps (500 ms) of neurons in each cluster. One-way ANOVA F $3,130=30.64 \mathrm{p}<0.0001$, Tukey's post hoc test $* \mathrm{p}<0.0001$. 
A

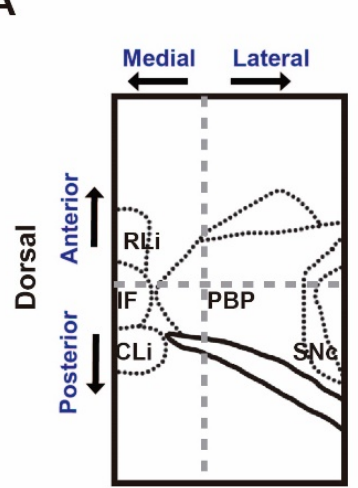

B

Cluster 1
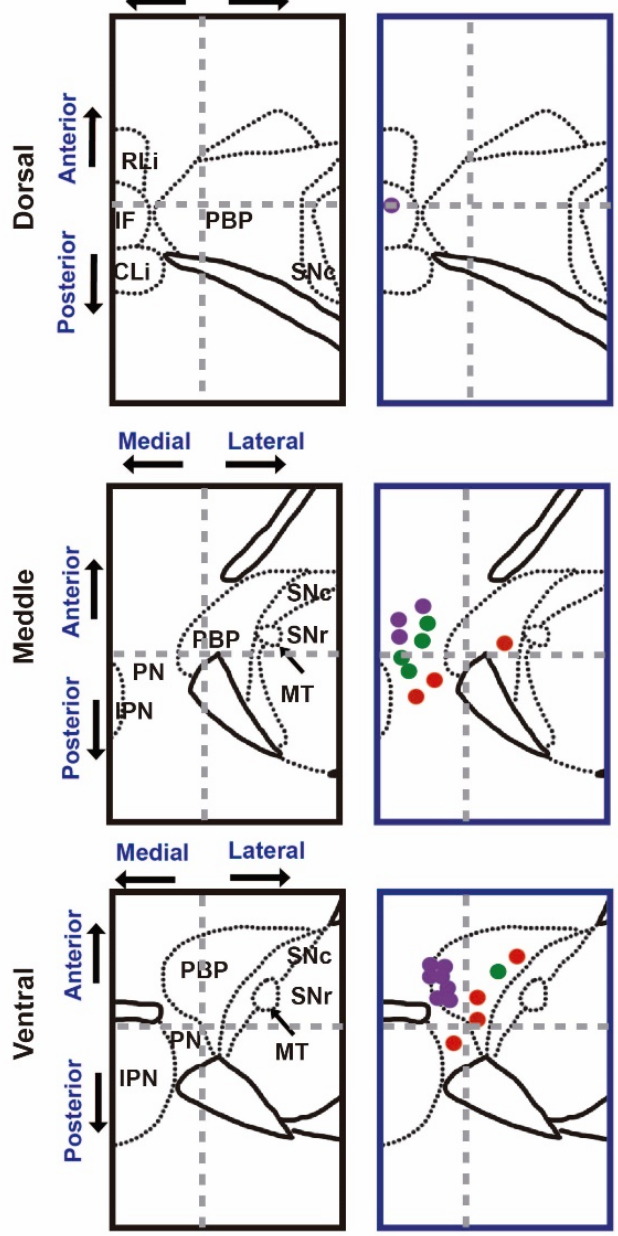

VGluT2+ VGaT+neurons
C

Cluster 2
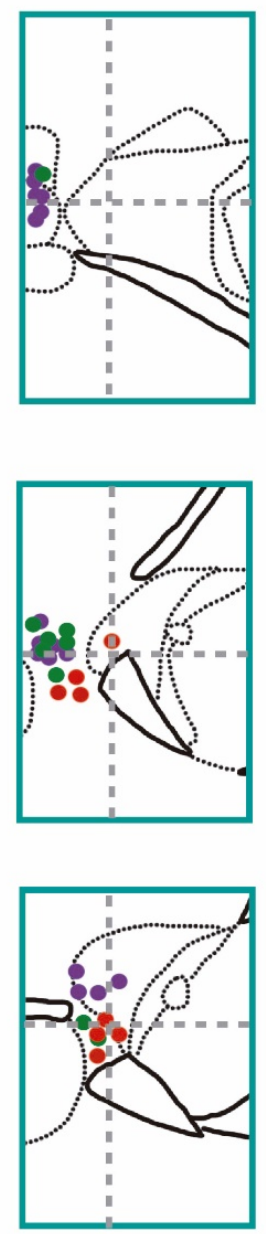

VGIuT2+ VGaT'neurons

D
Cluster 3
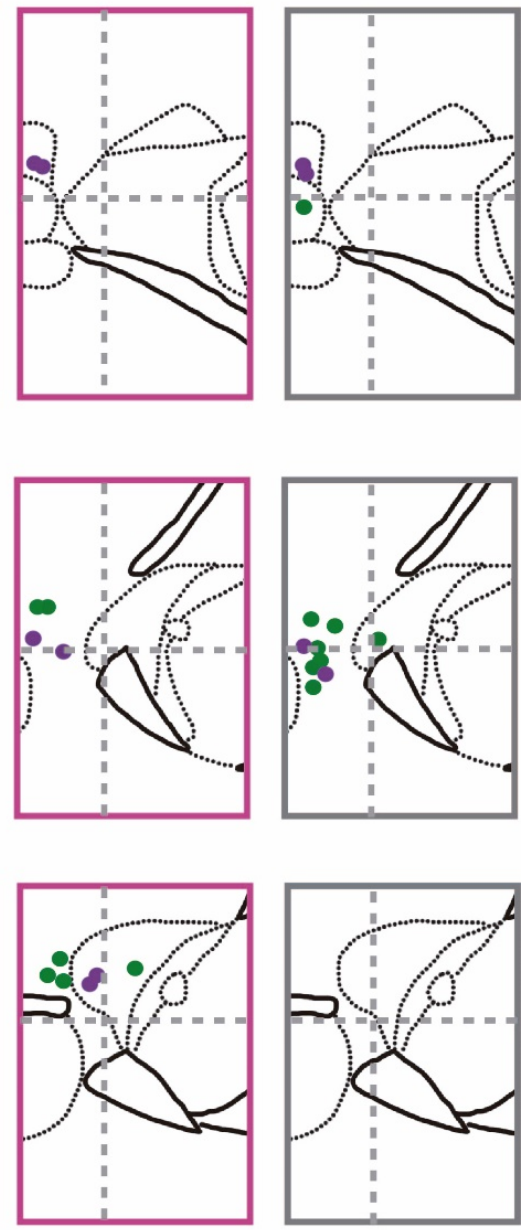

VGIuT2- VGaT+neurons

Figure 7. Topographic locations of VTA neurons by physiological cluster. (A) Schematic representations of dorsal, middle, and ventral horizontal VTA slices. (B-E) locations of recorded neurons from cluster 1 (B), cluster 2 (C), cluster 3 (D), and cluster 4 (E) in dorsal, middle, and ventral horizontal VTA slices. Grey dotted line divides the medial and lateral VTA. Each circle represents a single recorded neuron. 

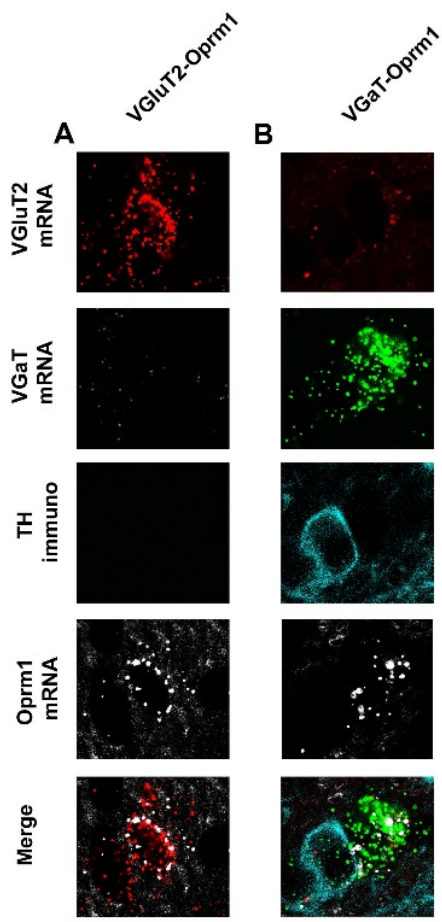

D

Expression of Oprm1 mRNA in different VTA cell types

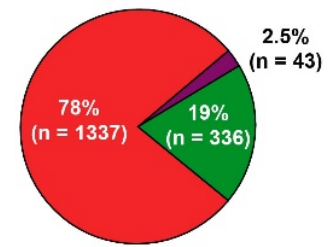

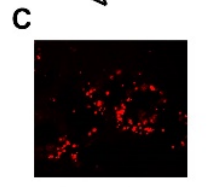
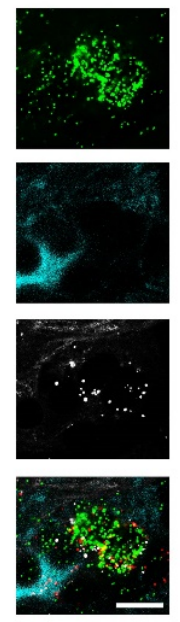

H
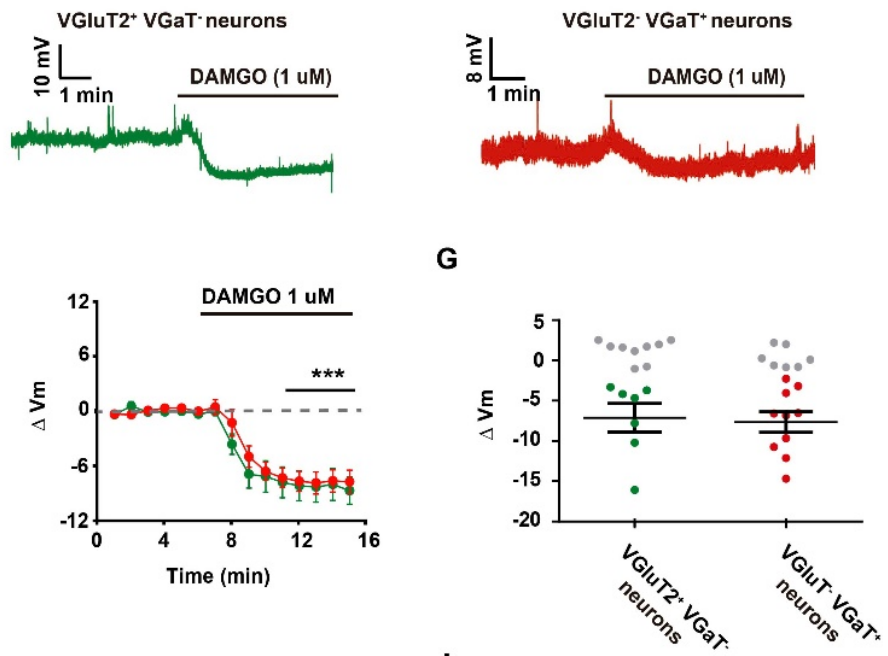

VGIuT2+ VGaT-neurons

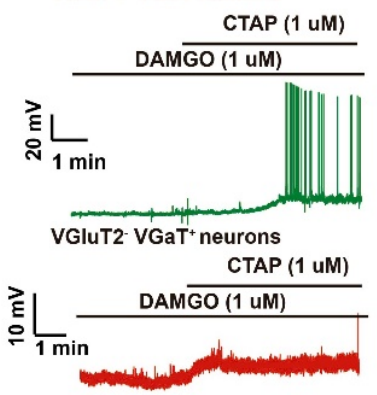

G

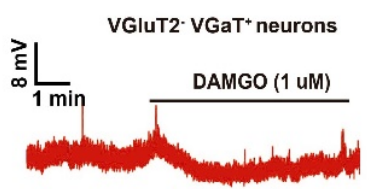

I

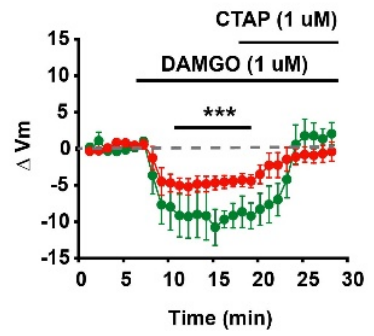

Figure 8. Functional MORs are present in VGluT2 ${ }^{+}$VGaT $^{-}$and VGluT2- VGaT $^{+}$VTA neurons. Detection of mRNA encoding VGluT2 (red), VGaT (green) or Oprm1 (white) and TH protein (cyan). (A) example neuron co-expressing VGluT2 and Oprm1 mRNAs. (B) example neuron co-expressing VGaT and Oprm1 mRNAs. (C) example neuron co-expressing VGluT2, VGaT, and Oprm1 mRNAs. (D) Percentage of VTA neurons expressing VGluT2 or VGaT mRNA with Oprm1 mRNA (3 mice). (E) Example current clamp traces from a VGluT2 $^{+}$VGaT $^{-}$(left) and a VGluT2 ${ }^{-} \mathrm{VGaT}^{+}$(right) neuron responsive to DAMGO bath application (F) Time course average showing-membrane potential of VGluT2 ${ }^{+} \mathrm{VGaT}^{-}$and VGluT2- $\mathrm{VGaT}^{+}$neurons in response to DAMGO. (G) Summary of DAMGO-induced changes in membrane potential in VGluT2 ${ }^{+}$VGaT $^{-}$and VGluT2$\mathrm{VGaT}^{+}$neurons. Responses in green and red circles and nonrespondes in grey circles. Paired $t$-test before and after DAMGO application $t_{6}=4.042 \mathrm{p}=0.0068$ for VGluT2 ${ }^{+}$VGaT $^{-}$neurons, $t_{10}=5.991 \mathrm{p}=0.0002$ for VGluT2 ${ }^{-}$ $\mathrm{VGaT}^{+}$neurons. (H) Example current clamp traces showing depolarizations in response to the $\mu$-opioid receptor selective antagonist CTAP in neurons that were hyperpolarized by DAMGO. (I) Time course of average membrane potential in neurons with application of DAMGO and CTAP. Repeated measures ANOVA $F_{2,14}=$ $15.0 \mathrm{p}<0.0003$, Dunnett's multiple comparison test $\mathrm{p}<0.0001$ for $\mathrm{VGluT}^{+} \mathrm{VGaT}^{-} ; \mathrm{F}_{2,17}=3.638 \mathrm{p}<0.0011$, Dunnett's multiple comparison test $\mathrm{p}<0.0001$ for VGluT2- $\mathrm{VGaT}^{+}$. 
A

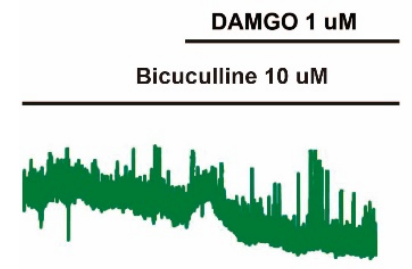

C

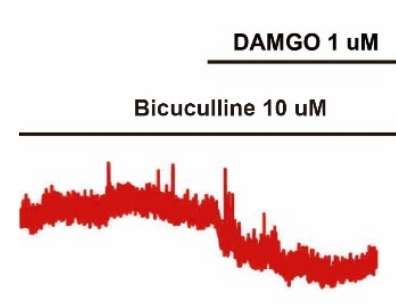

B

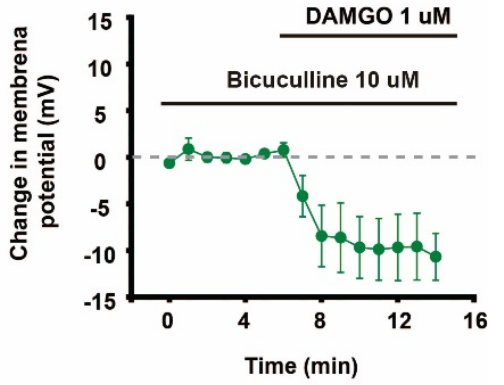

D

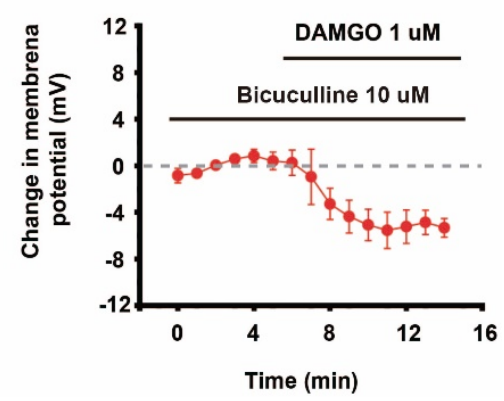

Figure 8-figure supplement 1. VGluT2 ${ }^{+} \mathrm{VGaT}^{+}$and VGluT2- $\mathrm{VGaT}^{+}$neurons are inhibited by DAMGO in the presence of $\mathbf{G A B A}$ receptor antagonist Bicuculline. (A) Example current clamp of a $\mathrm{VGluT}^{+} \mathrm{VGaT}^{-}$ neuron that responded to DAMGO in the presence of GABAA receptor antagonist Bicuculline. (B) Mean time course showing the change in membrane potential across VGluT2 ${ }^{+} \mathrm{VGaT}^{-}$neurons $(\mathrm{n}=4)$ to application of DAMGO in the presence of Bicuculline. (C) Example current clamp trace of a VGluT2- $\mathrm{VGaT}^{+}$neuron that responded to DAMGO in the presence of the $\mathrm{GABA}_{\mathrm{A}}$ receptor antagonist Bicuculline. (D) Mean time course of the membrane potential of VGluT2- $\mathrm{VGaT}^{+}$neurons in response to DAMGO in the presence of Bicuculline ( $\mathrm{n}=$ $5)$. 


\begin{tabular}{|c|c|c|c|c|c|c|c|c|}
\hline $\begin{array}{c}\text { Class of } \\
\text { VTA } \\
\text { neuron }\end{array}$ & $\begin{array}{c}\text { *Membran } \\
\text { e potential } \\
(\mathrm{mV})\end{array}$ & $\begin{array}{c}\text { Membran } \\
\text { e } \\
\text { resistance } \\
\text { (MOhms) }\end{array}$ & $\begin{array}{c}\text { Capacitanc } \\
\text { e (pF) }\end{array}$ & $\begin{array}{c}\text { *Rheobas } \\
\text { e (pA) }\end{array}$ & $\begin{array}{c}\text { AP } \\
\text { Threshold } \\
(\mathrm{mV})\end{array}$ & $\begin{array}{c}\text { Gap } \\
(\mathrm{mV})\end{array}$ & $\begin{array}{c}\text { AP } \\
\text { amplitud } \\
\text { e (mV) }\end{array}$ & $\begin{array}{c}{ }^{* A P} \\
\text { duration } \\
(\mathrm{ms})\end{array}$ \\
\hline $\begin{array}{c}\text { VGluT2 }^{+} \\
\text {VGaT }^{+} \\
(\mathbf{N}=\mathbf{5 2})\end{array}$ & $-68.0 \pm 1.2$ & $\begin{array}{c}644.7 \pm \\
68.4\end{array}$ & $30.9 \pm 2.0$ & 29.0 & $-39.1 \pm 0.5$ & 28.8 & 70.7 & $2.2 \pm 0.1$ \\
\hline $\begin{array}{c}\text { VGluT2 }^{+} \\
\text {VGaT }^{-} \\
(\mathrm{N}=48)\end{array}$ & $-64.6 \pm 0.9$ & $\begin{array}{c}655.7 \pm \\
42.5\end{array}$ & $26.6 \pm 1.3$ & $37.3 \pm 5.6$ & $-36.7 \pm 0.7$ & $28.0 \pm 1.0$ & $69.7 \pm 1.0$ & $2.9 \pm 0.2$ \\
\hline $\begin{array}{c}\text { VGluT2 }^{-} \\
\text {VGaT }^{+} \\
(\mathbf{N}=\mathbf{4 1})\end{array}$ & $-59.6 \pm 0.9$ & $\begin{array}{c}549.7 \pm \\
53.7\end{array}$ & $30.6 \pm 1.6$ & $14.9 \pm 2.9$ & $-38.4 \pm 0.8$ & $21.1 \pm 0.9$ & $72.9 \pm 1.6$ & $1.8 \pm 0.1$ \\
\hline
\end{tabular}

Table 1. Intrinsic membrane and action potential properties in VTA VGluT2 ${ }^{+} \mathrm{VGaT}^{+}, \mathrm{VGluT2}^{+} \mathrm{VGaT}^{-}$, and VGluT2- VGaT ${ }^{+}$neurons. Action potential (AP). Difference from membrane potential and action potential threshold (Gap). Millivolts (mV). Megaohms (MOhms). Picofarads (pF). Picoamperes (pA). Milliseconds (ms). Membrane potential: One-way ANOVA $F_{2,140}=16.65 \mathrm{p}<0.0001$; Tukey's pot hoc test ${ }^{*}$ VGluT2 $^{+}$VGaT $^{+}$vs VGluT2 $^{+}$VGaT $^{-} \mathrm{p}<0.05,{ }^{*}$ VGluT2 $^{+}$VGaT $^{-}$vs VGluT2 ${ }^{-}$VGaT $^{+}$p $<0.01,{ }^{* * *}$ VGluT2 $^{+}$ $\mathrm{VGaT}^{+}$vs VGluT2 ${ }^{-} \mathrm{VGaT}^{+} \mathrm{p}<0.001$. Rheobase: One-way ANOVA $\mathrm{F}_{2,140}=4.571 \mathrm{p}=0.0120$; Tukey's pot hoc test **VGluT2 ${ }^{+}$VGaT $^{-}$vs VGluT2- ${ }^{-} \mathrm{VGaT}^{+} \mathrm{p}<0.01$. Gap: One-way ANOVA $\mathrm{F}_{2,130}=12.37 \mathrm{p}<0.0001$; Tukey's pot hoc test ***VGluT2 ${ }^{+} \mathrm{VGaT}^{+}$vs VGluT2 ${ }^{-} \mathrm{VGaT}^{+} \mathrm{p}<0.001, * * * \mathrm{VGluT2}^{+} \mathrm{VGaT}^{-}$vs $\mathrm{VGluT2}^{-} \mathrm{VGaT}^{+} \mathrm{p}<$ 0.001. AP duration: One-way ANOVA F $2,140=17.26 \mathrm{p}<0.0001$; Tukey's pot hoc test $* * \mathrm{VGluT2}^{+} \mathrm{VGaT}^{+}$vs VGluT2 $^{+}$VGaT $^{-} \mathrm{p}<0.001,{ }^{* * *}$ VGluT2 $^{+}$VGaT $^{-}$vs VGluT2- ${ }^{-}$VGaT $^{+} \mathrm{p}<0.00$. 